\title{
Stacked autoencoders as new models for an accurate Alzheimer's disease classification support using resting-state EEG and MRI measurements
}

\author{
Raffaele Ferri $^{\mathrm{a}, 1, *}$, Claudio Babiloni ${ }^{\mathrm{b}, \mathrm{c}, 1}$, Vania Karami ${ }^{\mathrm{d}}$, Antonio Ivano Triggiani ${ }^{\mathrm{e}}$, Filippo Carducci ${ }^{\mathrm{b}}$, \\ Giuseppe Noce $^{\mathrm{f}}$, Roberta Lizio ${ }^{\mathrm{f}}$, Maria T. Pascarelli ${ }^{\mathrm{a}}$, Andrea Soricelli ${ }^{\mathrm{f}, \mathrm{g}}$, Francesco Amenta ${ }^{\mathrm{d}}$, \\ Alessandro Bozzao ${ }^{\mathrm{h}}$, Andrea Romano ${ }^{\mathrm{h}}$, Franco Giubilei ${ }^{\mathrm{h}}$, Claudio Del Percio ${ }^{\mathrm{b}}$, Fabrizio Stocchi ${ }^{\mathrm{i}}$, \\ Giovanni B. Frisoni ${ }^{\mathrm{j}, \mathrm{k}}$, Flavio Nobili ${ }^{\mathrm{l}, \mathrm{m}}$, Luca Patanè ${ }^{\mathrm{n}}$, Paolo Arena ${ }^{\mathrm{o}}$
}

${ }^{a}$ Department of Neurology I.C., Oasi Research Institute - IRCCS, Troina, Italy

b Department of Physiology and Pharmacology "V. Erspamer", Sapienza University of Rome, Rome, Italy

${ }^{\mathrm{c}}$ Hospital San Raffaele Cassino, Cassino (FR), Italy

${ }^{\mathrm{d}}$ Department of Pharmaceutical Sciences and Health Products, University of Camerino, Camerino, Italy

e Department of Clinical and Experimental Medicine, University of Foggia, Foggia, Italy

${ }^{\mathrm{f}}$ IRCCS SDN, Napoli, Italy

${ }^{\mathrm{g}}$ Department of Motor Sciences and Healthiness, University of Naples Parthenope, Naples, Italy

${ }^{\mathrm{h}}$ Department of Neuroscience, Mental Health and Sensory Organs, Sapienza University of Rome, Rome, Italy

${ }^{\mathrm{i}}$ IRCCS San Raffaele Pisana, Rome, Italy

${ }^{\mathrm{j}}$ LENITEM (Laboratory of Epidemiology, Neuroimaging and Telemedicine), IRCCS Centro "S. Giovanni di Dio-F.B.F.", Brescia, Italy

${ }^{\mathrm{k}}$ Memory Clinic and LANVIE - Laboratory of Neuroimaging of Aging, University Hospitals and University of Geneva, Geneva, Switzerland

${ }^{1}$ Clinica Neurologica, IRCCS Ospedale Policlinico San Martino, Genova, Italy

${ }^{\mathrm{m}}$ Dipartimento di Neuroscienze, Oftalmologia, Genetica, Riabilitazione e Scienze Materno-infantili (DiNOGMI), Università di Genova, Italy

${ }^{\mathrm{n}}$ Dipartimento di Ingegneria, Università degli Studi di Messina, Messina, Italy

${ }^{\circ}$ Dipartimento di Ingegneria Elettrica, Elettronica e Informatica, University of Catania, Catania, Italy

See Article, pages 207-209

\section{A R T I C L E I N F O}

\section{Article history:}

Accepted 11 September 2020

Available online 15 October 2020

\section{Keywords:}

Alzheimer's Disease (AD)

Resting State Electroencephalography (rsEEG)

Low-resolution brain electromagnetic tomography (LORETA)

Stacked Artificial Neural Networks (ANNs)

with Autoencoders

\section{H I G H L I G H T S}

- Artificial neural networks with stacked autoencoders detected Alzheimer's dementia patients based on EEG and structural MRI variables.

- Classification accuracies over control participants reached 80\% (EEG), 85\% (MRI), and 89\% (both).

- These results motivate future multi-centric, harmonized prospective and longitudinal crossvalidation studies.

\begin{abstract}
A B S T R A C T
Objective: This retrospective and exploratory study tested the accuracy of artificial neural networks (ANNs) at detecting Alzheimer's disease patients with dementia (ADD) based on input variables extracted from resting-state electroencephalogram (rsEEG), structural magnetic resonance imaging (sMRI) or both. Methods: For the classification exercise, the ANNs had two architectures that included stacked (autoencoding) hidden layers recreating input data in the output. The classification was based on LORETA source estimates from rsEEG activity recorded with 10-20 montage system (19 electrodes) and standard sMRI variables in 89 ADD and 45 healthy control participants taken from a national database.

Results: The ANN with stacked autoencoders and a deep leaning model representing both ADD and control participants showed classification accuracies in discriminating them of $80 \%, 85 \%$, and $89 \%$ using rsEEG, sMRI, and rsEEG + sMRI features, respectively. The two ANNs with stacked autoencoders and a deep leaning model specialized for either ADD or control participants showed classification accuracies of $77 \%, 83 \%$, and $86 \%$ using the same input features.
\end{abstract}

\footnotetext{
* Corresponding author.

E-mail address: rferri@oasi.en.it (R. Ferri).

1 These two Authors gave the same contribution to the development of the present study.
} 
Conclusions: The two architectures of ANNs using stacked (autoencoding) hidden layers consistently reached moderate to high accuracy in the discrimination between ADD and healthy control participants as a function of the rsEEG and sMRI features employed.

Significance: The present results encourage future multi-centric, prospective and longitudinal crossvalidation studies using high resolution EEG techniques and harmonized clinical procedures towards clinical applications of the present ANNs.

() 2020 International Federation of Clinical Neurophysiology. Published by Elsevier B.V. All rights reserved.

\section{Introduction}

In aging, the most prevalent progressive neurodegenerative dementing disorder is Alzheimer's Disease (AD), which induces progressive cognitive deficits, loss of autonomy/independence in activities of everyday, and behavioral symptoms (Braak and Braak, 1995).

The US National Institute on Aging-Alzheimer's Association (NIA-AA) and the International Working Group (IWG) have recommended the use of the following in-vivo biomarkers for the assessment of AD patients in clinical research studies (Förstl and Kurz, 1999; Dubois et al., 2014; Albert et al., 2011; Jack et al., 2010, 2018; McKhann et al., 2011; Sperling et al., 2011). For diagnostic purposes, measures of amyloidosis and tauopathy in the brain can be extracted from cerebrospinal fluid (CSF) or maps of positron emission tomography (PET), while measures of AD-related neurodegeneration or synaptic dysfunction tauopathy can be derived from structural magnetic resonance imaging (sMRI) and ${ }^{18}$ Fluorodeoxyglucose-PET (Förstl and Kurz 1999; Dubois et al. 2014; Karami et al., 2018; Jicha, 2011; Jack et al., 2018; Ottoy et al., 2019).

The above biomarkers are valid and reliable but partially or totally invasive and relatively expensive for screening millions of old seniors with cognitive deficits and risk of $\mathrm{AD}$ (Rossini et al., 2020). Therefore, research studies have been testing noninvasive and cost-effective techniques including the spectral analysis of resting state electroencephalographic (rsEEG) rhythms, especially at delta $(<4 \mathrm{~Hz})$, theta $(4-8 \mathrm{~Hz})$, and alpha $(8-12 \mathrm{~Hz})$ frequency bands (Babiloni et al., 2020a,b). Those rhythms mainly reflect partially overlapping neurophysiological oscillatory mechanisms of synchronization of neural activity in thalamocortical, corticothalamic, and corticocortical circuits involved in the regulation of vigilance in quiet wakefulness (Babiloni et al., 2020a,b).

In the past decades, investigations in $\mathrm{AD}$ patients with dementia (ADD) and amnesic mild cognitive impairment (ADMCI) have reported that rsEEG may be promising disease neurophysiological markers. In relation to normal elderly (Nold) subjects, groups of ADD patients showed abnormally high power of widespread delta and theta rhythms, as well as low power of posterior alpha and/or beta $(13-30 \mathrm{~Hz})$ rhythms (Dierks et al., 1993, 2000; Huang et al., 2000; Jeong, 2004; Ponomareva et al., 2003).

In the review by Jonkman (1997), 16 qualified rsEEG studies, appeared in 1983-1995, reported a classification accuracy ranging from $54 \%$ to $100 \%$ (median of $81 \%$ ) in the discrimination between Nold and ADD individuals. In another review with more stringent selection criteria (Jelic and Kowalski, 2009), 46 qualified rsEEG studies appeared until 2008 reported a classification accuracy ranging from $80 \%$ to $85 \%$ (median of $81 \%$ ) in the discrimination between ADD patients (or MCI) and control subjects (Nold and patients with other dementing disorders).

Recently, we have developed a research line to evaluate the hypothesis that Nold and ADD individuals may be classified with at least a moderate accuracy (70-80\%) using rsEEG source activities estimated by the standard LORETA toolbox (Pascual-Marqui et al.,
1994). Specifically, LORETA source estimates of rsEEG rhythms were used to derive a single variable - occipital delta/alpha ratio - that allowed a classification accuracy of $75 \%$ in the discrimination of 120 ADD patients and 100 matched Nold seniors by the computation of receiver operating characteristic (ROC) curves (Babiloni et al., 2016). In another study, we re-analyzed that database giving 4 LORETA source estimates (i.e. delta/alpha 1 and theta/alpha 1 ratios in posterior cortical lobes) as an input to resilient backpropagation ANNs, confirming a moderate classification accuracy of 77\% (Triggiani et al., 2017).

In the framework of that research line, the present retrospective and exploratory study aimed at testing three novel hypotheses based on the use of LORETA source estimates. The first hypothesis was that more advanced ANNs such as those based on autoencoders (AEs) may produce a classification accuracy $>80 \%$ in the discrimination between Nold and ADD individuals by LORETA source estimates. The second hypothesis was that based on these ANNs, the classification accuracy between Nold and ADD individuals may be similar using LORETA source estimates and standard SMRI markers of brain neurodegeneration. The third hypothesis was that the combined use of those LORETA source estimates and SMRI markers may produce the best discrimination accuracy in this exercise.

The study was designed to compare performances of two different architectures and learning processes of autoencoder-based ANNs for testing the hypothesis of moderate to high classification accuracies of both architectures in the discrimination between Nold and ADD individuals based on rsEEG and sMRI markers. The confirmation of this hypothesis may indicate the general robustness of the present methodological approach.

To achieve the study aim, we used a national database of clinical, rsEEG, and sMRI data in 45 Nold seniors and 89 ADD patients, including 16 Nold (35\%) and 27 ADD (30\%) individuals who already participated in our previous study by Triggiani and colleagues (Triggiani et al., 2017). Of note, a previous study of an independent research group successfully used autoencoder-based ANNs in the discrimination ( $>80 \%$ of accuracy) between Nold, ADD, and ADMCI individuals by a combination of relatively invasive or expensive markers of AD derived from SMRI, PET, and CSF in Alzheimer's Disease Neuroimaging Initiative (ADNI) cohorts (Suk et al., 2015).

\section{Materials and methods}

\subsection{Subjects}

The clinical, rsEEG, and sMRI data of the present study refer to 89 ADD and 45 Nold subjects, matched for age, education years, and gender. Local institutional ethics committees have endorsed the information collection and the analysis for scientific reasons. In accordance with the World Medical Association Code of Ethics (Declaration of Helsinki), each participant or caregiver subscribed a written informed consent.

Probable ADD was diagnosed based on the criteria of the Diagnostic and Statistical Manual of Mental Disorders, fourth edition 
(DSM-IV-TR; American Psychiatric Association) and the National Institute of Neurological Disorders and Stroke-Alzheimer Disease and Related Disorders (NINCDS-ADRDA) working group (McKhann et al., 1984). Individuals underwent medical, neuropsychological, neurological, and psychiatric evaluations including Instrumental Activities of Daily Living scale (IADL; Lawton and Brod, 1969), Mini-Mental State Examination (Folstein et al., 1975), Clinical Dementia Rating (CDR; Hughes et al., 1982), and Geriatric Depression Scale (GDS; Yesavage et al., 1982). Criteria for exclusion included any kind of proof of other types or causes of dementia such as frontotemporal dementia (The Lund and Manchester Group, 1994), vascular dementia diagnosed on the basis of the National Institute of Neurological Disorders and Stroke and Association Internationale pour la Recherché et l'Enseignement en Neurosciences (NINDS-AIREN) working group (Román et al., 1993), Parkinson disease (PD; Gelb et al., 1999), Dementia with Lewy Bodies (DLB; McKeith et al., 2005), metabolic syndrome, nutritional deficits, tumors, etc. When before EEG recordings, given, benzodiazepines, antidepressant and/or antihypertensive were temporally stopped for about $24 \mathrm{~h}$. This procedure did not guarantee that a full washout of the drug-longer periods would not have been valid for apparent ethical reasons-but it made it possible for patients with ADD to compare the drug situation. Note that most ADD patients ( $>95 \%$ ) followed a long-term therapy with normal daily doses of acetylcholinesterase inhibitors including donepezil, rivastigmine, or galantamine in standard dosages. The Nold subjects were evaluated medically, neurologically, and psychiatrically including Mini Mental Stage Evaluation (MMSE; Folstein et al., 1975), Clinical Dementia Rating (CDR; Hughes et al., 1982) and Geriatric Depression Scale (GDS; Yesavage et al., 1982), to exclude topics with a history of neurological or psychiatric disorders (including substances abuse) from the research. Finally, according to the Alzheimer's Disease Neuroimaging Initiative (ADNI; http://adni.loni.usc.edu), another exclusion criterion was a score below 27 for the Nold topics at the MMSE and above 24 for the AD topics.

Table 1 summarizes the subjects' population and some clinical information. T-tests assessed age, education, MMSE score, and Individual Alpha Frequency peak (IAFp; see below for a description of this index) differences ( $p<0.05$, one-tailed) between groups (Nold and ADD).

For the MMSE score $(p<0.0001$; higher MMSE score in the Nold than in the ADD group) and for the IAFp ( $p<0.0001$; higher IAF in the Nold than in the ADD group), as expected, there was no statistically significant difference in age, gender, and education $(p>0.05)$.

\subsection{EEG recordings and preliminary processing of data}

As a premise, this retrospective and exploratory study is based on experiments performed in the framework of different clinical studies over time, so some methodological procedures were equal or very similar across the participating clinical units, while others

Table 1

Demographic and clinical characteristics of the subjects; mean \pm standard deviation.

\begin{tabular}{lll}
\hline Subjects & Nold $(\mathrm{n}=45)$ & $\mathrm{AD}(\mathrm{n}=89)$ \\
\hline Gender (Female/Male) & $30 / 15$ & $53 / 36$ \\
Age & $70.55 \pm 7.31$ & $72.5 \pm 8.42$ \\
Education (years) & $9.43 \pm 4.59$ & $8.3 \pm 4.5$ \\
MMSE score & $29 \pm 1.49$ & $18.5 \pm 3.16$ \\
IAF & $9.35 \pm 1.08$ & $8.13 \pm 1.54$ \\
\hline
\end{tabular}

Nold: normal elderly subjects; AD: Alzheimer's Disease; MMSE score: Mini Mental Stage Evaluation; IAF: Individual Alpha Frequency for individual analysis of rsEEG frequency and band power; rsEEG: resting-state electroencephalogram. showed some differences. Furthermore, EEG procedures used in the present study are based on some assumptions and the results must be interpreted as related to those assumptions. Specifically, we followed the procedures of preliminary rsEEG data analysis used in previous investigations of our research group to enrich the interpretation of the present results (Babiloni et al., 2016; Triggiani et al., 2017).

Instructions to participants for the rsEEG recordings were very similar in all clinical units. All subjects were kindly asked to stay in a relaxed state with the eyes closed during the experiments. They were also kindly asked not to move or talk and keep the mind wandering without focused mentalization. During the experimental recordings, the experimenters noted down, in the experimental sheet: drowsiness, eyes opening, gentle verbal warnings given to the participants when signs of drowsiness, significant muscle tensions or movements appeared, and hand and/or head movements or other confounding events disturbing EEG recordings.

In all experiments, at least 5 minutes of electrophysiological data were recorded by professional digital EEG systems licensed for clinical applications (i.e., EB-Neuro Be-light, Micromed, Brain vision, etc.) from 19 exploring scalp electrodes placed according to the 10-20 montage system (i.e., Fp1, Fp2, F7, F3, Fz, F4, F8, T3, C3, Cz, C4, T4, T5, P3, Pz, P4, T6, O1, and O2). The ground electrodes were placed in the posterior midline, while the reference electrodes were located in different positions across participating clinical units (i.e., linked earlobes, mastoids, vertex, etc.) in relation to local standard protocols and trials. This difference did not affect the present findings as the LORETA toolbox produces cortical source estimates independent of the placement of ground and reference electrodes. In other words, it provides the identical EEG source estimate using any placement of ground and reference electrodes (Pascual-Marqui et al., 1994).

During the rsEEG recordings, electrode impedances were kept below $5 \mathrm{KOhm}$. The rsEEG recordings were performed using $128 \mathrm{~Hz}$ or higher sampling rate $(128-1,024 \mathrm{~Hz})$ with an antialiasing bandpass between $0.01 \mathrm{~Hz}$ and $60-100 \mathrm{~Hz}$.

In parallel to the rsEEG recording, bipolar vertical and horizontal electrooculographic (EOG) signals and one-channel electrocardiographic (ECG) signals were also acquired using the same sampling frequency adopted to record the rsEEG data (128$1,024 \mathrm{~Hz}$ ). Consequently, rsEEG, EOG, and ECG signals had the same sampling rate, so EOG and ECG signals could be used for the artifact detection and their off-line correction when adequate.

As mentioned above, some rsEEG datasets were recorded using a relatively low sampling frequency of $128 \mathrm{~Hz}(<20 \%$ of the rsEEG datasets collected). It should be remarked that such a sampling frequency is sub-optimal for an ideal reconstruction of rsEEG signal beyond $40 \mathrm{~Hz}$ without aliasing. Ideally, a factor of 3-4 between the low-band pass limit and the rsEEG sampling frequency should be set (Burgess, 2019).

In the preliminary analysis, the rsEEG data were split into 2-s epochs and analyzed off-line centrally. This segmentation allowed the use of standard toolboxes for the spectral analysis of rsEEG activity such as Fast Fourier Transform (FFT) and eLORETA source estimation, which assume the stationary of rsEEG activity. Furthermore, it allowed to minimize the rejection of rSEEG data around a short of period of artifactual activity. The use of those procedures allowed a better understanding of the present results in light of previous reference evidence of our research line (Babiloni et al., 2016; Triggiani et al., 2017), but it implied the focus on the linear components of rsEEG signals for the classification purposes.

Two independent experimenters (G. N. and R. L.) performed a visual analysis of EOG and rsEEG data blind to the clinical diagnosis associated with the electrophysiological datasets. They rejected those with artifacts due to instruments, electronic noise, headneck movements, and face muscle tension. They also rejected 
rsEEG epochs with amplitude values exceeding $100 \mu \mathrm{V}$. Particular attention was given to the contamination of ocular activity (i.e., blinking) on electrophysiological data recorded at frontal (i.e., F7, F3, Fz, F4, and F8) and frontopolar (Fp1 and Fp2) electrodes. This specific exam was based on the comparison of EOG and rsEEG traces. The rsEEG epochs with artifacts marked as eye movements and blinking were given as inputs to a software toolbox based on an autoregressive model for their possible correction. Technical details and performances of this procedure were reported elsewhere (Moretti et al., 2003). Noteworthy, the outcome of this procedure was visually revised by the two experimenters (G. N. and R. L.). All Nold and ADD datasets showed less than $20 \%$ of artifact-free rsEEG epochs, without significant differences between the Nold and ADD groups ( $t$-test, $p>0.05$ ).

To harmonize rsEEG data recorded using different reference electrodes and sampling frequency rates, artifact-free rsEEG epochs were off-line frequency-band passed at $0.1-45 \mathrm{~Hz}$ and down sampled, when appropriate, to make the sampling rate of all artifact-free rsEEG datasets in the Nold and ADD participants equal to $128 \mathrm{~Hz}$. For sake of harmonization of all datasets, the recorded rsEEG data were re-referenced to the common average reference.

Spectral power density of rsEEG rhythms was computed by Fast Fourier Transform (FFT, Welch algorithm, Hanning window, no phase shift) using $0.5 \mathrm{~Hz}$ as frequency resolution. According to previous rsEEG studies carried out in Nold and ADD seniors (Babiloni et al., 2005, 2006, 2011, 2013; Jelic et al., 1996; Besthorn et al., 1997; Chiaramonti et al., 1997), the following standard frequency bands were considered: delta $(2-4 \mathrm{~Hz})$, theta $(4-8 \mathrm{~Hz})$, alpha 1 (8-10.5 Hz), alpha $2(10.5-13 \mathrm{~Hz})$, beta $1(13-20 \mathrm{~Hz})$, beta 2 (20-30 Hz), and gamma (30-40). The contiguous frequency bands shared a bin of frequency in line with the assumption of partially overlapping neurophysiological oscillatory mechanisms (Klimesch, 1999; Babiloni et al., 2020b).

\section{3. rsEEG rhythms cortical sources computation by LORETA}

To estimate cortical source activity (i.e., neural current density) from rsEEG rhythms, the official LORETA toolbox (Pascual-Marqui et al., 1994; http://www.unizh.ch/keyinst/NewLORETA/LORETA01.htm) was used. It solves the EEG linear inverse problem estimating neural currents in 2,394 voxels with $7 \mathrm{~mm}$ of spatial resolution that form the spherical cortical source model of the LORETA toolbox. The other two compartments of the head model are those representing electrical properties of scalp and skull. Those voxels of the cortical comportment are co-registered to the Talairach probability brain atlas developed in the Brain Imaging Center of the Montreal Neurological Institute (Talairach and Tournoux, 1988).

As EEG inverse problem solutions are typically underdetermined and unconditioned due to the greater number of unknown samples (current density at each voxel) than scalp electrodes, LORETA toolbox regularizes inverse solutions selecting the maximally smooth source activity solution explaining input scalprecorded EEG data (Pascual-Marqui et al., 1994).

We used the LORETA toolbox to estimate rSEEG cortical source activities in all 2,394 voxels from 1 to $45 \mathrm{~Hz}$. Afterward, we normalized LORETA source estimates by the following procedure. For each participant, we averaged LORETA source estimates across all voxels and frequency bins from 1 to $45 \mathrm{~Hz}$. This averaged value served as a denominator to normalize (i.e., ratio) LORETA source estimates at all cortical voxels and for all frequency bins. This normalization method typically fits rsEEG power density into a Gaussian distribution and decrease its variability between subjects (Nuwer, 1997; Leuchter et al., 1993). After the above normalization, the LORETA solutions lost the original physical dimension and are represented by arbitrary units. In the normalized scale, the value " 1 " at all frequencies $(0.5-45 \mathrm{~Hz}$ ) and cortical volume voxels was equivalent to the mean of the dipole current density.

In line with the LORETA intrinsic low-spatial resolution of its solutions, we averaged the normalized LORETA source estimates for bilateral ROIs representing cortical lobes of the head model. Specifically, the LORETA solution for a given lobar ROI was obtained averaging the normalized LORETA source estimates across all the voxels of that ROI. The bilateral lobar ROIs were the following: (pre)frontal, central (i.e., Brodmann areas 6, 4, 3, 2, and 1), parietal (except Brodmann areas 3, 2, and 1), temporal, occipital, and limbic (Table 2).

\subsection{The rsEEG features used as inputs for ANNS}

In total, we used 42 rsEEG features as inputs for the present ANNs. They are listed in the following: LORETA source current density in the parietal, temporal, occipital, limbic, central, and frontal ROIs systematically combined with delta, theta, alpha 1 , alpha 2 , beta 1 , beta 2 , and gamma frequency bands. For example, the following 6 rsEEG source estimates (features) were extracted for the delta band: "frontal delta”, "central delta”, "parietal delta”, “occipital delta", "temporal delta”, and "limbic delta."

\subsection{Recording and analysis of sMRI data}

Proton density, T1- and T2-weighted sMRIs were acquired based on the standard research settings mostly by 1.5 or 3.0 Tesla scanners at the following clinical neuroimaging centers based on local neuroimaging scientific platforms: Sapienza University of Rome (Italy), IRCCS Fatebenefratelli of Brescia (Italy), IRCCS San Raffaele Pisana of Rome (Italy), IRCCS SDN of Naples (Italy), IRCCS Oasi Maria SS of Troina (Italy), and Service of Neurophysiopathology of the University of Genova (Italy). Some of these units (IRCCS SDN of Naples; Sapienza University of Rome and Service of Neurophysiopathology of the University of Genova) provided the MRIs based on the ADNI protocol (http://www.adni-info.org/). All 1.5 Tesla MRI scanners used harmonized sequences and scan parameters for image acquisition. In the same line, all 3.0 Tesla MRI scanners used harmonized sequences and scan parameters for image acquisition.

The centralized analysis of all MRIs was performed by the group of the Department of Physiology and Pharmacology "Vittorio Erspamer" at Sapienza University of Rome. The MRI scans were visually checked to confirm that they were free from structural defects or technical artifacts. The MRI scans were also checked as general quality of the images, signal-to-noise ratio, and wellbalanced number of MRI datasets at 1.5 and 3.0 Tesla in the Nold and ADD participants to allow a combined use of 1.5 and 3.0 Tesla MRIs as inputs to ANNs for classification purposes.

The analysis of MRIs was conducted using the Functional Magnetic Resonance Imaging of the Brain (FMRIB) Software Library

Table 2

List of the used Brodmann areas of the cortical sources of rsEEG (resting-state electroencephalogram) rhythms by LORETA software to define the ROIs (regions of interest).

\begin{tabular}{ll}
\hline Regions of interest & LORETA Brodmann Areas \\
\hline Frontal & $8,9,10,11,44,45,46,47$ \\
Central & $1,2,3,4,6$ \\
Parietal & $5,7,30,39,40,43$ \\
Temporal & $20,21,22,37,38,41,42$ \\
Occipital & $17,18,19$ \\
Limbic & $31,32,33,34,35,36$ \\
\hline
\end{tabular}

rsEEG: resting-state electroencephalogram. 
(FSL), to identify the probability that a given voxels under the skull may belong to gray matter (GM), white matter (WM) or cerebrospinal fluid (CSF). Specifically, the following method estimated the probability that a given voxel may belong to GM. First, the FSLBET pipeline conducted an atlas-based parceling operation to segment subject's brain regions from MRIs automatically. This was followed by a single-channel segmentation using the FAST tool of FSL. It produced a-posterior probability maps in the space of the individual subject for GM, WM, and CSF, with each voxel in the range $0-1$ where 1 is the maximum probability (Zhang et al., 2001). FAST is specifically based on Hidden Markov Random Field (HMRF) model and a related Expectation Maximization (EM) algorithm (Zhang et al., 2001). This HMRF model has benefits regarding the manner that MRI spatial information is encoded through nearby voxels, which leads to spatial regularization and decreasing noise impact on the segmentation. The HMRF model is integrated by an EM algorithm to obtain precise and robust segmentations (Zhang et al., 2001). In order to fit and provide a standard labeled template, non-linearly normalizations (FSL-FNIRT tool) were computed for the determination of the GM probability. The output of this procedure was an associated spatial transformation matrix from the subject's space to a standard template space. This model was based on averaged high-resolution MRIs obtained from the subjects of this research, which included anatomical channels (T1-, T2-, and proton density-weighted images), tissue channels (CSF probability, GM probability, WM probability, and tissue labels), and two cortical parcellation maps, namely the TZO map, using the model of Tzourio-Mazoyer et al. (2002), and the socalled LPBA40 map, based on the LONI Probabilistic Brain Atlas of 40 subjects (Willis-Shattuck et al., 2008).

Based on this parcellation of the MRIs, the following 16 sMRI markers were obtained and used as inputs for the AE ANNs: volume of the Total Intracranial Volume (TIV; $\left.\mathrm{cm}^{3}\right), \mathrm{GM}\left(\mathrm{cm}^{3}\right), \mathrm{WM}$ $\left(\mathrm{cm}^{3}\right)$, CSF under the skull $\left(\mathrm{CSF} ; \mathrm{cm}^{3}\right)$, left hippocampus (LHippo; $\mathrm{cm}^{3}$ ), left hippocampus normalized to the TIV (LHippoTiv), left hippocampus normalized to the GM (L-HippoGM), right hippocampus (R-Hippo; $\mathrm{cm}^{3}$ ), right hippocampus normalized to the TIV (R-HippoTIv), right hippocampus normalized to the GM (R-HippoGM), total hippocampus (T-Hippo; $\mathrm{cm}^{3}$ ), total hippocampus normalized to the TIV (T-HippoTiv), total hippocampus normalized to the GM (T-HippoGM), cortical GM (Cortex; $\mathrm{cm}^{3}$ ), cortical GM normalized to the TIV (CortexTiv), and cortical GM normalized to the total GM (CortexGM). Of note, we measured both volume (i.e., number of voxels) and concentration (ratio by TIV) of gray matter to be sensitive to complementary and informative dimensions of the AD-related neurodegenerative process in the cerebral cortex and hippocampus to improve the detection of ADD patients (Weiner et al., 2017; Lee et al., 2020).

\subsection{ANN architecture and procedures}

Fig. 1 depicts a block diagram summarizing the two architectures of the present ANNs (i.e., SAE and AEs) developed for the classification between the Nold and ADD individuals based on the sMRI and/or rsEEG features of the input datasets (i.e., the "patterns"). In some classification trials, the input datasets included only SMRI features. In other classification trials, they included only rsEEG features or a combination of the rsEEG and sMRI features.

A diagram of the adopted SAE is reported in Fig. 2A. These ANNs had an input layer receiving those input features, two stacked autoencoding hidden layers recreating input data in the output, and a softmax output layer (SAE ANN) or a layer for the computation of the distribution of the reconstruction error (AE ANNs) to produce the classification outcome (Liao et al., 2015).

Referring to Fig. 2A, the information of the input features represented in the first autoencoding hidden layer ( $\mathrm{H} 1$ in Autoencoder

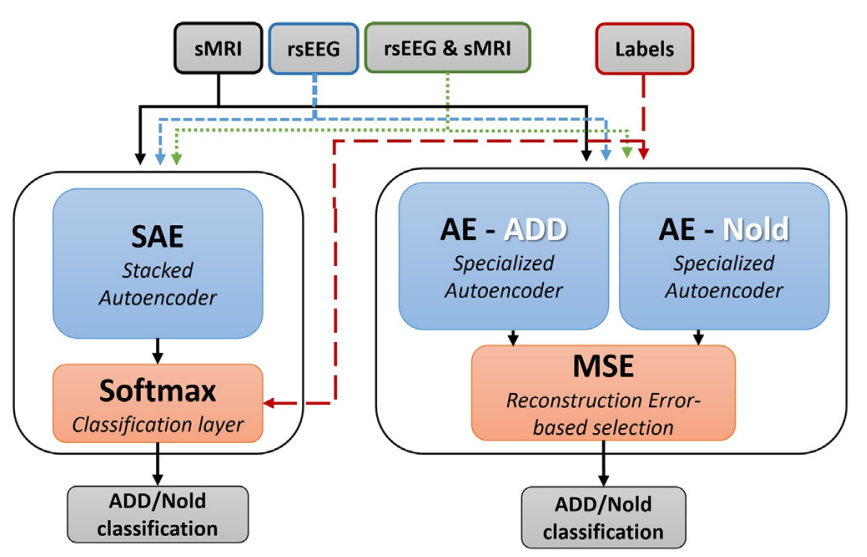

Fig. 1. Block scheme of two developed architectures for Alzheimer's disease patients with dementia (ADD) vs Nold classification used in the present study: a stacked autoencoder (SAE) with a softmax output layer and a pair of internal specialized AEs with an output layer based on the reconstruction error (MSE). Three different input datasets were considered including sMRI and resting state electroencephalographic (rsEEG) features, separately and together. The labels indicating the correct output class are used in both schemes but at different levels of the processing steps. Nold: normal elderly subjects; SAE: stacked autoencoder; sMRI: structural magnetic resonance imaging.

A) was used as an input for the second autoencoder (Autoencoder B) creating a new hidden representation encoded into $\mathrm{H} 2$. This iteratively compresses the feature dimensional representation space containing all needed information for the reconstruction. The hidden layers $\mathrm{H} 1$ and $\mathrm{H} 2$ build up the SAE, which contains a softmax output layer which reformulates the hidden layers representation into a probability distribution, to provide the classification of the individual dataset of rsEEG and/or sMRI features as "Nold" or "ADD".

The second approach consists in training separately two AEs, each one specialized for reconstructing only one of the two classes. As depicted in Fig. 2B, during the testing phase, the two AEs are tested with all the testing patterns, and their outputs are processed in a comparison layer, where the class selection is chosen according to the minimum of the reconstruction error (MSE) computed separately by the two networks specialized for "Nold" or "ADD".

Overall, in the classification procedure, the "low-level" sMRI and rsEEG features of the input datasets were used to derive a "high-level" representation of latent features by the deep learning using the stacked autoencoding hidden layers.

To optimize their structure and parameters, the present ANNs (i.e., SAE and AEs) were subjected to a learning process developed in two phases: 1) the pre-training of the stacked autoencoding hidden layers to achieve optimum original parameters in a greedy layer-wise manner and 2) the main training allowing the fine tuning of the deep network to choose the optimum parameters across all the mentioned layers.

In the pre-training phase, the learning process in the two autoencoding hidden layers exploited an optimization algorithm based on the scaled conjugate gradient descent (Møller, 1993) and did not use the class label (i.e., "Nold" or "ADD") of the available patterns (i.e., the datasets with the selected sMRI and/or rsEEG features). In the learning process of the softmax layer, the class label was, instead, used to categorize the output feature vectors autoencoding hidden layers. As mentioned above, the outcome of the learning process in the deep network was the probability of an input pattern to be classified as ADD vs. Nold. It must be noticed that the initialization of the network weights via this pre-training phase makes different the deep network with respect to a conventional ANN, reducing the problem of local minima (Larochelle et al., 2009). 


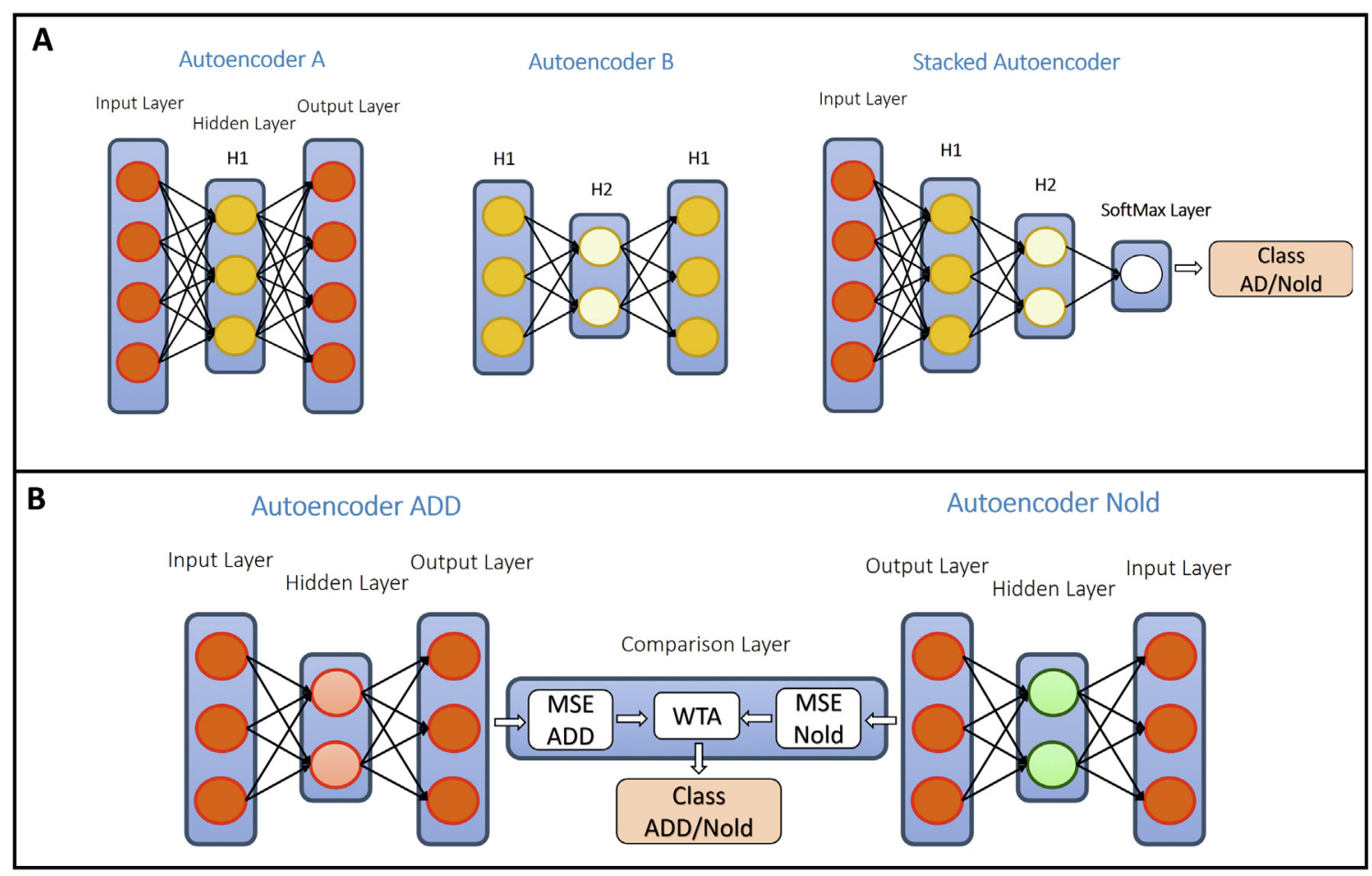

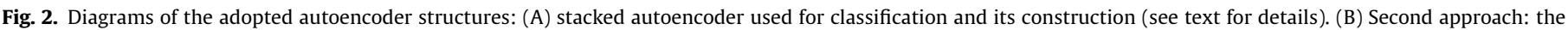

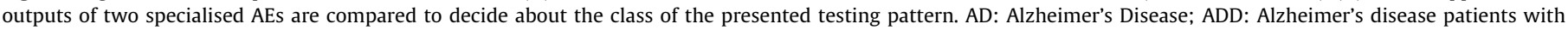
dementia; AE: autoencoder; MSE: reconstruction error; Nold: normal elderly subjects.

In the main training phase, the results obtained for the deep neural network were improved by performing a further learning phase on the whole multi-layer network. This phase stacked the input layer together with the two autoencoding hidden layers and the final classification layer. In this phase, the fine tuning of the network was performed on the training data using a supervised learning approach. The learning algorithm adopted to optimize the entire deep network was the gradient descent back propagation. Summarizing, in this learning process, sparse learning of the enhanced feature vectors (i.e., a concatenation of the initial lowlevel features and the stacked autoencoding hidden layerslearned features) was used to select the regressed features of targets effectively, namely the clinical diagnosis of Nold or ADD.

The mentioned main training phase was developed in two steps. In the first step, the best number of the artificial neurons in the two autoencoding hidden layers was defined based on systematic simulation trials and the generation of classification performance maps. Specifically, the simulation trials were performed changing systematically the number of the artificial neurons in the first (from 5 to 25 with step 5) and the second (from 2 to 8 with step 2) autoencoding hidden layers. The activation function adopted for the neurons was the logistic sigmoid function (i.e., logsig). In this exercise, the following three learning parameters were optimized. The first parameter was the "iteration", namely the numbers of models created and used to develop the performance statistics. For each model, the topology and learning parameters remained unchanged, while the division of the individual datasets (i.e., Nold and ADD individuals associated with rsEEG and/or sMRI features) in learning (80\%) and testing (20\%) patterns was differently randomized, performing a parameter optimization through internal cross-validation. The second parameter was called "MaxEpoches", namely the maximum number of learning epochs used for the autoencoding hidden layers and the softmax layer. The third parameter was called "MaxEpoches Stacked", namely the maximum number of learning epochs used for a fine-tuning of the complete stacked network. As a criterion of best classification performance, we considered both the mean classification accuracy in the testing phase and the standard deviation of that accuracy across the iterations. Ideally, the best number of artificial neurons in the two autoencoding hidden layers was producing the highest classification accuracy combined with the lowest standard deviation.

Of note, the optimization of the "iteration", "MaxEpoches", and "MaxEpoches Stacked" in the simulation trials must be considered as one of the most important aspects of the procedure. Considering the relatively high number of rsEEG and/or sMRI input features and the relatively limited number of individual Nold and ADD datasets associated with those features, an important challenge in the learning process of the deep network model was to avoid the overfitting of the learning datasets. The risk of this overfitting increases when the learning error in the training phase become too low, thus predicting a poor generalization capability of the ANN to classify independent testing datasets (not used in the training phase) correctly. To tackle this risk, we optimized the above three parameters in the simulation trials to balance the learning errors in fitting the learning patterns and the classification accuracy in the discrimination of the testing patterns. In line with previous evidence (Lawrence and Giles, 2000), the key solution to mitigate the overfitting of the training patterns was the use of a relatively low number of learning epochs for the fine tuning of the networks. As an outcome, the optimal setting of the three parameters for the SAE was 500 iterations, 250 MaxEpoches, and 10 MaxEpoches Stacked. Furthermore, that setting for the SAE was 500 iterations and 20 MaxEpochs.

In the second step, each network was trained using the learning parameters optimized as mentioned above to determine the connection weights among all the neurons in the layers. These weights had the functionality to optimize the association between the individual sMRI and/or rsEEG features, coded as an input in the autoencoding hidden layers, and the correct classification in the output 
neurons as "Nold" or "ADD". To this purpose, we used new training datasets (i.e., Nold and ADD individuals) of sMRI and/or rsEEG features not used in the first step of the procedure. As an outcome of this second step, a sparse learning on the augmented feature vectors, i.e. a concatenation of the original low-level features and the stacked autoencoding hidden layers-learned features, was applied to select features that efficiently regressed the targets, namely the clinical diagnosis (i.e. Nold or ADD).

Finally, we used or fused the selected sMRI and rsEEG features via ANNs for the classification of testing individual datasets as "Nold" or "ADD". In this phase of the classification exercise, the network performance was evaluated in the discrimination between the Nold and ADD individual datasets using a testing subset never used in this experiment.

The performance of the ANNs was expressed as percentages (\%) by the following indices:

(1) Sensitivity, defined as the ADD rate of the test datasets properly categorized as $A D$; this index was referred as True Positive (TP) or TP rate (TPR).

(2) Specificity, defined as the Nold rate of the testing datasets correctly classified as Nold; this index was called as True Negative (TN) or TN rate (TNR).

(3) Accuracy, defined as the sum of the TP and the TN divided by the all number of the datasets including the two classes (ADD and Nold).

This step-by-step classification process with optimization of the ANNs, training phase, and testing phase was repeated 500 times (iterations). Any iteration resulted in different values of sensitivity, specificity, and accuracy. The final reports in the "Results" section referred to the average sensitivity, specificity, and accuracy values over all 500 iterations.

In general, the above procedure was valid for the two classification architectures used in the present study (i.e., SAE and AE). In the first model, the training phase of the SAE was performed with both Nold and ADD feature datasets as input to the unique stacked network. In the second architecture, a pair of AEs were trained with Nold and ADD feature datasets, separately. The testing datasets were given to both specialized AEs, and the AE with the lower mean squared reconstruction error was considered as the best classification. If the $\mathrm{AE}$ trained with the Nold (ADD) feature datasets produced the lowest reconstruction error, the individual dataset was associated with a Nold (ADD) individual.

The above classification procedure was repeated three times for the optimization of the networks specific for the classification of the 42 rsEEG features, the 16 sMRI features, and 58 rsEEG + sMRI features, respectively.

\section{Results}

Considering the proposed architecture, the first step performed was associated with identification of the optimal number of hidden-layer neurons. The adopted performance index is the classification accuracy, and a series of maps were reproduced for the three different subsets of input features. Fig. 3 depicts the classification performance maps for the SAE network, illustrating the topology of the selected structures, on the basis of the input features, as reported in Table 3.

The same procedure was adopted for the classification architecture based on the specialized $\mathrm{AE}$, and the obtained results indicate

\section{Table 3}

Best performing SAE (stacked autoencoder) network topology optimized based on the classification accuracy as depicted in Fig. 2. Depending on the selected input features the optimal number of hidden neurons was defined.

\begin{tabular}{ll}
\hline Input features & Network topology \\
\hline rsEEG & $42-20-6-2$ \\
SMRI & $16-10-8-2$ \\
rsEEG \& sMRI & $58-20-4-2$
\end{tabular}

rsEEG: resting-state electroencephalogram; sMRI: structural magnetic resonance imaging.

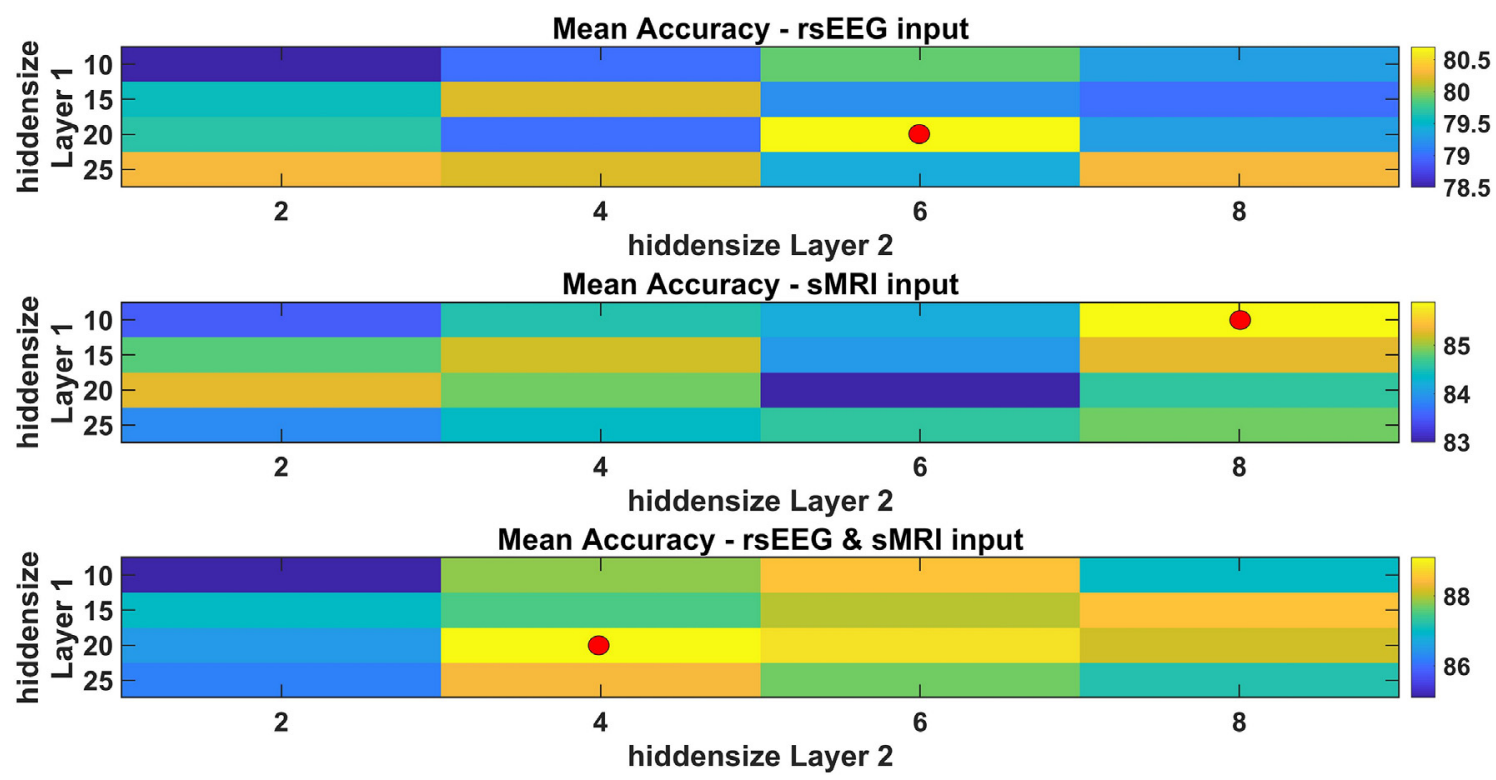

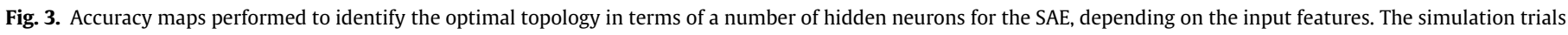

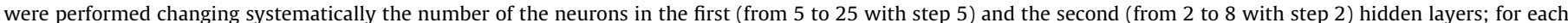

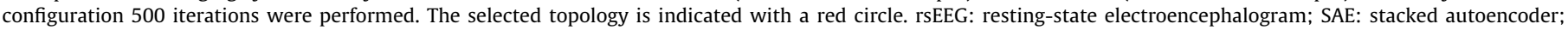
sMRI: structural magnetic resonance imaging. 
that the performances change very slightly. Therefore, we considered the same topology with 10 hidden neurons for both $\mathrm{AE}$, independently from the selected input features.

Based on the SAE topology optimized with the first step of the procedure, the two levels of training and the testing phases were performed. The classification rate obtained adopting this procedure is reported in Table 4: for the classification of the 42 rsEEG features, we observed a sensitivity of $89 \%$, a specificity of $64 \%$, and an accuracy of $80.3 \%$; for the classification of the 16 sMRI features, we observed a sensitivity of $90 \%$, a specificity of $76 \%$, and an accuracy of $85 \%$; finally, for the classification of the 58 rsEEG-sMRI features, we observed a sensitivity of $93.3 \%$, a specificity of $81 \%$, and an accuracy of $89 \%$.

Table 4

Classification performance for the SAE (stacked autoencoder) depending on the input features. The mean percentage \pm standard deviation of sensitivity, specificity, and accuracy, over 500 iterations are reported.

\begin{tabular}{llll}
\hline Input features & Sensitivity & Specificity & Accuracy \\
\hline rsEEG & $88.9 \pm 8.0$ & $64.1 \pm 15.9$ & $80.3 \pm 7.1$ \\
SMRI & $89.4 \pm 7.5$ & $75.9 \pm 15.3$ & $84.6 \pm 6.9$ \\
rSEEG \& sMRI & $93.3 \pm 6.8$ & $81.0 \pm 12.9$ & $89.1 \pm 5.8$ \\
\hline
\end{tabular}

rsEEG: resting-state electroencephalogram; sMRI: structural magnetic resonance imaging.
The learning procedure, followed for the SAE, is fundamental to obtain this performance. In particular, the role of the fine tuning of the whole network is crucial as demonstrated in Fig. 4, where the learning and testing performances, using all the 58 features, are reported comparing the results obtained during the first and second learning phase. Similar behavior is also verifiable, considering a reduced input space.

The results obtained for the SAE were compared with the second architecture with specialized AE. The classification rate obtained adopting this model is reported in Table 5. For the classification of the 42 rsEEG features, we observed a sensitivity of $86.5 \%$, a specificity of $58 \%$, and an accuracy of $77 \%$. For the classification of the 16 sMRI features, we observed a sensitivity of $78.8 \%$, a specificity of $89.7 \%$, and an accuracy of $82.4 \%$. Finally, for the classification of the 58 rsEEG-SMRI features, we observed a sensitivity of $92.3 \%$, a specificity of $74.2 \%$, and an accuracy of $86.2 \%$.

Fig. 5 shows the distribution of the MSE for the ADD and Nold $\mathrm{AE}$ comparing learning and testing phases. Adopting the criterion above (maximization of the accuracy over the testing datasets) and selecting "MaxEpoch" equal to 20 (see "Methods" for more detail), the learning and testing Gaussian shape distributions were similarly arranged over the error axis. Smaller values in the learning epochs may have led to larger training errors, whereas larger ones may have caused overfitting of the learning datasets

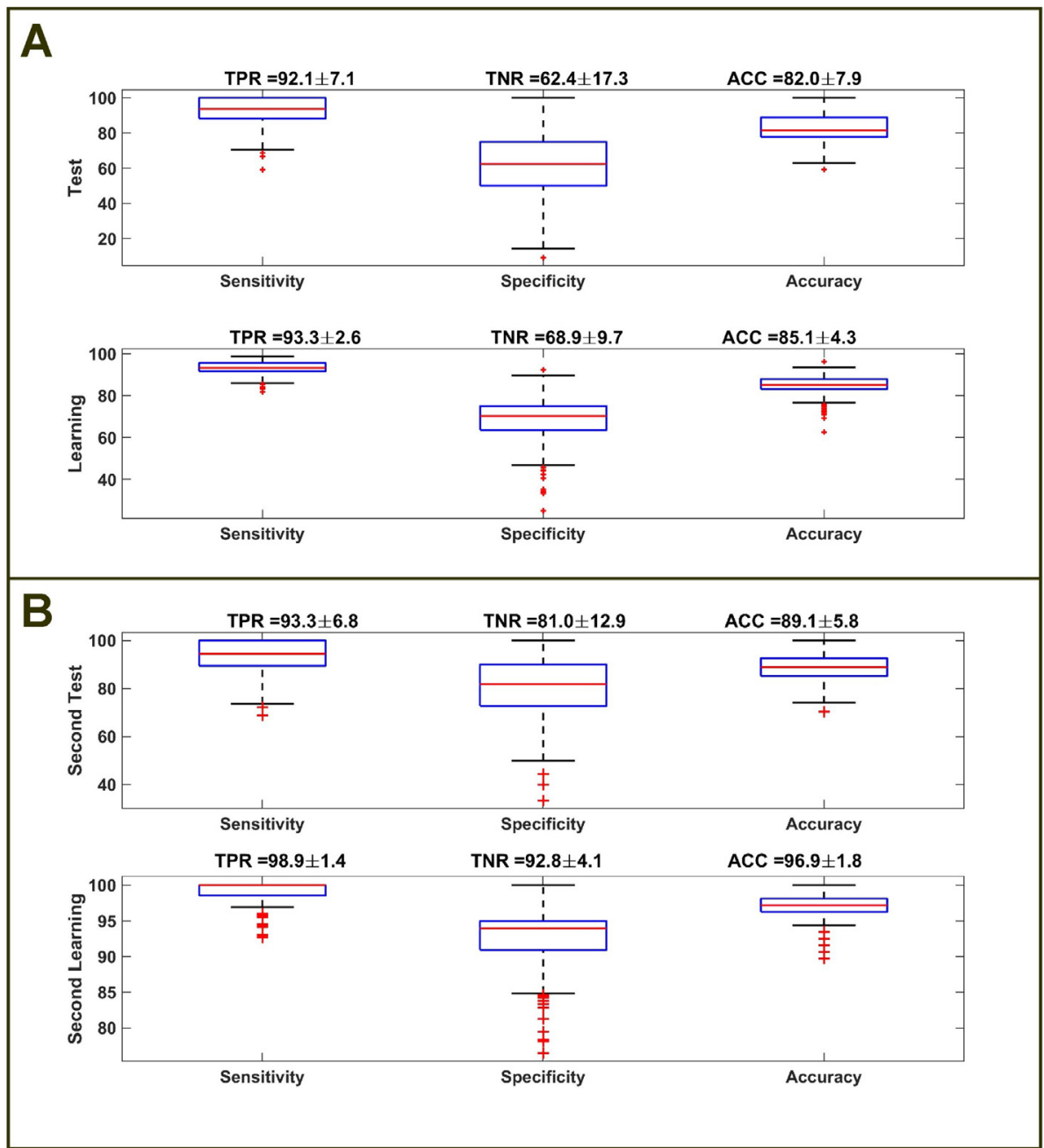

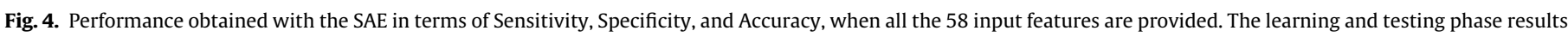

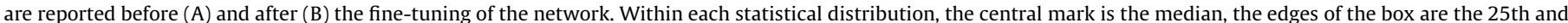

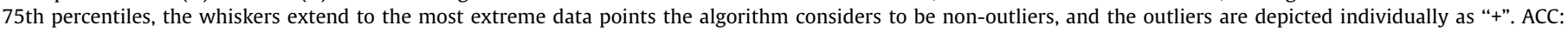
Accuracy; SAE: stacked autoencoder; TNR: true negative rate; TPR: true positive rate. 
Table 5

Classification performance for the specialized $\mathrm{AE}$ (autoencoder) depending on the input features. The mean percentage \pm standard deviation of sensitivity, specificity, and accuracy, over 500 iterations are reported.

\begin{tabular}{llll}
\hline Input features & Sensitivity & Specificity & Accuracy \\
\hline rsEEG & $86.5 \pm 11.2$ & $58.0 \pm 17.3$ & $77.0 \pm 7.3$ \\
SMRI & $78.8 \pm 10.6$ & $89.7 \pm 15.3$ & $82.4 \pm 7.3$ \\
rSEEG \& sMRI & $92.3 \pm 6.9$ & $74.2 \pm 14.2$ & $86.3 \pm 6.0$ \\
\hline
\end{tabular}

rsEEG: resting-state electroencephalogram; sMRI: structural magnetic resonance imaging.

(patterns) significantly affecting the error distributions between learning and testing (classification accuracy of testing datasets) patterns.

The performance obtained comparing learning and testing phase using Sensitivity, Specificity, and Accuracy, when all the 58 input features were provided, is reported in Fig. 6. This performance presents some similarities with the results reported in Fig. 5, related to the SAE network, in terms of the statistical distribution of the performance indexes.

To test the discriminant value of those rsEEG and sMRI variables at the group level, a control analysis was performed. We compared those rsEEG and sMRI variables between the groups of Nold and ADD participants as follows.

Concerning the rsEEG part, the normalized LORETA source activities (dependent variable) were compared by an ANOVA design the exploratory statistical threshold of $p<0.01$. This ANOVA used three factors such as Group (Nold and ADD), Band (delta, theta, alpha 1 , alpha 2 , beta 1 , beta 2 , and gamma), and ROI (frontal, central, parietal, temporal, occipital, and limbic). The individual variability of the alpha power peak in the spectrum was considered by the variable called individual alpha frequency peak (IAFp; Klimesch, 1999). For each subject, the IAFp was defined as the frequency bin showing the maximum global power density in the range between 6 and $13 \mathrm{~Hz}$, averaging the power density values across all scalp electrodes (Klimesch, 1999). Results showed that the mean IAFp was higher in frequency in the Nold group ( $9.4 \mathrm{~Hz}, \pm 0.2$ standard error of the mean, SE) than the ADD group $(8.0 \mathrm{~Hz}, \pm 0.2 \mathrm{SE})$ as revealed by a $t$-test $(p=0.000005)$.

Fig. 7 shows the grand average of the normalized rsEEG source activities (i.e., regional normalized LORETA solutions) relative to a statistically significant ANOVA interaction effect $(F=16,7$; $p<0,00001$ ) among the factors Group, Band, and ROI. The IAFp was used as a covariate. A Bonferroni correction was applied for 7 bands X 6 ROIs $=42(p<0.05 / 42=0.001)$. In the figure, the normalized rsEEG source activities exhibit marked differences between the Nold and ADD groups. As compared to the Nold group, the ADD group shows that the rsEEG source activities at the alpha 1 and alpha 2 bands are generally smaller, confirmed by the post-hoc Duncan test $(p<0.000005)$. The differences at the alpha 1 and alpha 2 bands are observed in the posterior ROIs including the parietal, temporal, occipital, and limbic lobes $(p<0.000005)$. As compared to the Nold group, the ADD group also shows greater normalized rsEEG source activities at the delta band. This effect was confirmed by the post-hoc Duncan test in the frontal, central, parietal, temporal, and occipital lobes ( $p<0.001-0.00001)$.

Overall, the results of the above control analysis suggest that present LORETA-based rsEEG source activities showed marked differences between the Nold and ADD groups as a solid basis for their use as an input for the ANN classification exercise.

Concerning the sMRI part of the control analysis, the standard biomarkers used for the classification exercise were compared between the Nold and ADD groups by univariate Kruskal-Wallis $\mathrm{H}$ tests using with the exploratory statistical threshold of $p<0.01$ (one tail predicting signs of greater atrophy in the ADD group than the Nold group). These sMRI biomarkers comprised what follows: white matter (WM) hyperintensity, gray matter (GM) volume normalized by the total intracranial volume (TIV), left hippocampus volume, left hippocampus volume normalized by the TIV, right hippocampus volume, right hippocampus normalized by the TIV, left hippocampus volume normalized by the GM, total hippocampus volume, total hippocampus volume normalized by the TIV, total hippocampus volume normalized by the GM, cerebral cortex volume, cerebral cortex volume normalized by the TIV, and cerebral cortex volume normalized by the GM. Results showed that most of those sMRI biomarkers exhibited statistically significant abnormalities in the ADD group as compared to the Nold group ( $p<0.01-0.001$ ). These abnormalities pointed to a remarkable atrophy in the ADD group in practically all variables representing cerebral cortex and hippocampus (Table 6).

Overall, the results of the above control analysis suggest that present LORETA-based rsEEG source activities showed marked differences between the Nold and ADD groups as a solid basis for their use as an input for the ANN classification exercise.
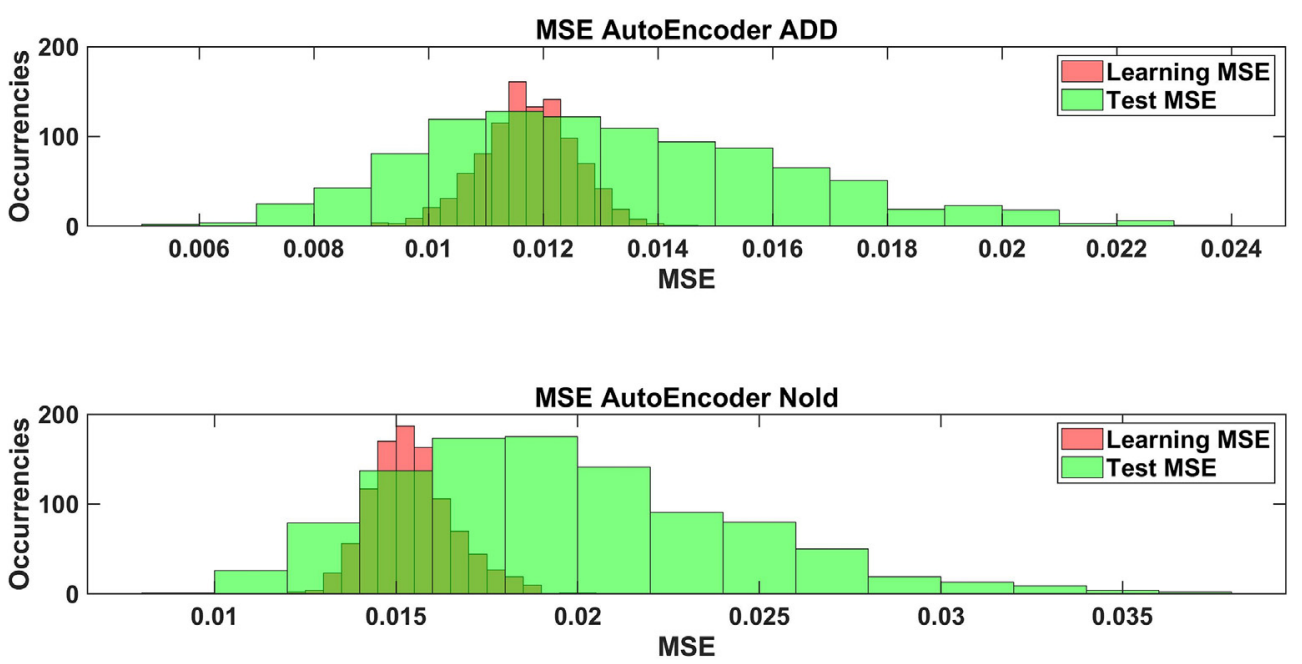

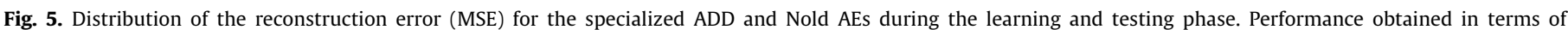

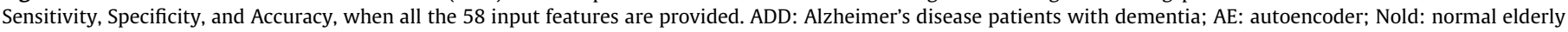
subjects. 


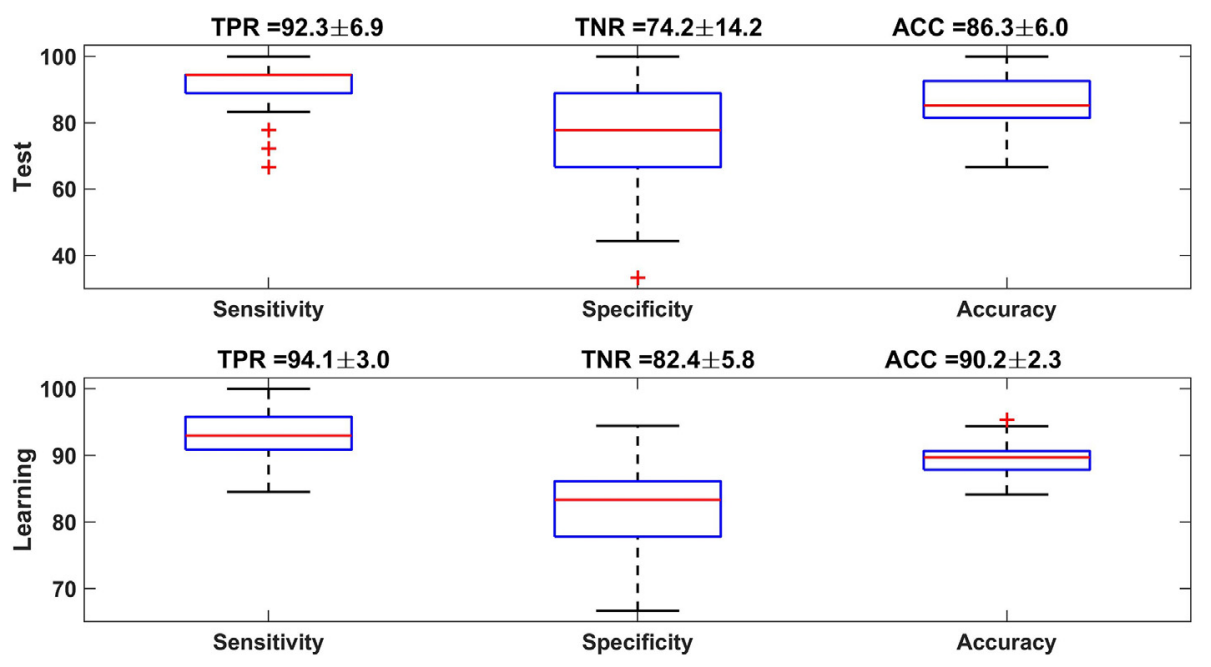

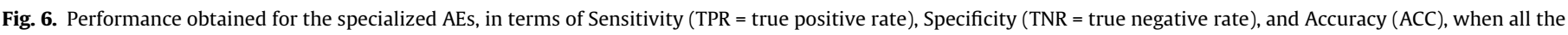

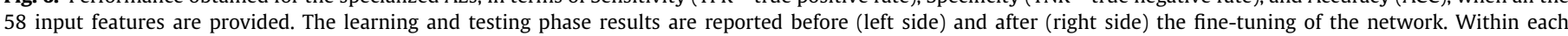

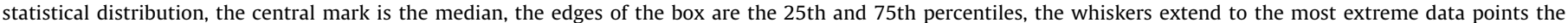
algorithm considers to be non-outliers, and the outliers are depicted individually as "+". AE: autoencoder.

\section{Discussion}

In a previous study, resilient backpropagation ANNs have been reported to reach $77 \%$ of accuracy in classifying ADD vs. Nold individuals using (LORETA) rsEEG cortical source activities as input features for the classification (Triggiani et al., 2017). Here we used another class of ANNs with stacked autoencoding hidden layers to: $1)$ obtain higher classification accuracy $(>80 \%)$ in the discrimination between Nold and ADD individuals by LORETA source estimates and 2) compare the classification accuracy of LORETA source estimates in relation to standard SMRI markers of brain neurodegeneration and a combination of LORETA source estimates and those sMRI markers. Two different architectures and learning processes of autoencoder-based ANNs were used and performances compared to evaluate the general robustness of the present methodological approach.

Results showed that the ANN with the SAE architecture achieved a moderate-to-high accuracy in the classification between the Nold and ADD individuals based on 42 rsEEG (LORETA) source features spanning over several frequency bands and cortical lobes. Specifically, there was a moderate-to-high classification accuracy (i.e., 80-89\%) of the SAE architecture of the ANN in the discrimination between the Nold and ADD individuals as a function of the rsEEG (i.e., 80\%) and sMRI (i.e., 85\%) variables considered separately, with the best findings obtained when those variables were combined together (i.e., 89\%). Interestingly, the ANNs with both SAE and AE architectures exhibited a slightly better (i.e., 2-3\%) classification accuracy using sMRI as compared to rsEEG variables (see Tables 4 and 5), thus indicating the robustness of their outcome.

Results also showed higher (i.e. 5\%) classification accuracies by the SAE over the EA architecture in the present ANNs. Based on this finding, there would be a certain advantage in the sue of an ANN with the SAE architecture able to represent and separate two clinically relevant classes (i.e., Nold and ADD) of patterns in its internal deep learning model. In that ANN, the SAE deep learning model developed in the present study may be directly tested in the discrimination between Nold seniors and patients with prodromal stages of the disease such as ADMCI status without further learning processes. Of course, this advantage would be concrete only with the maintenance of moderate to high classification accuracies even in the discrimination between Nold and ADMCI individuals. We expect this similarity based on the prediction that $\mathrm{AD}$ neuropathology is quite significant in ADMCI patients and may produce rsEEG and sMRI features qualitatively like those found in ADD patients (Babiloni et al., 2017, 2018). Differently, the discrimination between Nold and ADMCI individuals with ANNs based on AEs would imply the construction and learning of another deep learning model specialized in the detection of ADMCI patients. This additional work would have the advantage to specialize that autoencoder-based ANN based on rsEEG and sMRI markers derived from ADMCI patients.

These results are in agreement with earlier evidence of our group; for example, our resilient backpropagation ANNs produced a discriminant accuracy between Nold and ADD individuals of $77 \%$ using 4 (LORETA) rsEEG source activity as features (i.e. occipital, temporal, and parietal theta/alpha; occipital delta/alpha; Triggiani et al., 2017). Furthermore, univariate classifications by the ROC curves reported accuracies of $75-80 \%$ based on LORETA occipital alpha sources or the ratio between parietooccipital delta and alpha source activities (Babiloni et al., 2015, 2016; Lizio et al., 2016).

The present results also extend earlier evidence of other groups showing that rsEEG scalp-sensor features allow a mild discrimination between Nold and ADD individuals of about 80\% (Brenner et al., 1986, 1988; Claus et al., 1999; Hooijer et al., 1990; Strijers et al., 1997). To achieve an accuracy of $84 \%$ in the classification between Nold and ADD individuals, and 78\% between ADD and MCI individuals, Huang et al. (2000) did mix alpha and theta global field power. Furthermore, Adler et al. (2003) reported that in the classification of Nold and ADD individuals, the left temporal alpha coherence and the global theta power density returned an accuracy of $80 \%$. Moretti et al. (2011) showed that enhanced global theta/ gamma and alpha $3 /$ alpha 2 power density ratios resulted in an accuracy of $88 \%$ in the prediction from MCI to ADD or non-ADD. Trambaiolli et al. (2011) reported that the temporal energy modulation in the delta, theta, alpha, beta, and gamma bands resulted in an accuracy of $91 \%$ in the classification of Nold and ADD individuals. Engedal et al. (2015) reported that 20 rsEEG markers (including the alpha frequency peak, total power density, and coherence between electrodes) allowed an accuracy of $90 \%$ in the discrimination between ADD patients and seniors without or with other forms of dementing disorders (e.g., Dementia with Lewy Bodies). More information of this literature can be discovered in two 


\section{STATISTICAL ANOVA INTERACTION AMONG GROUP, BAND, AND ROI}

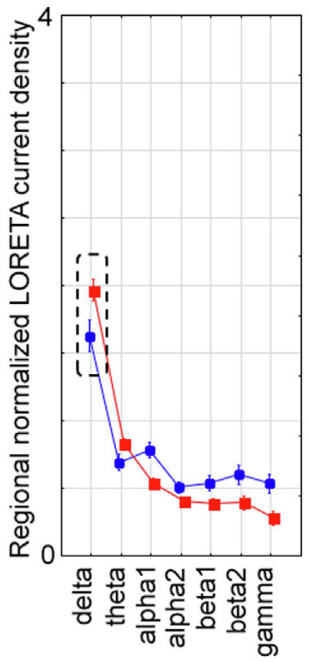

Frontal

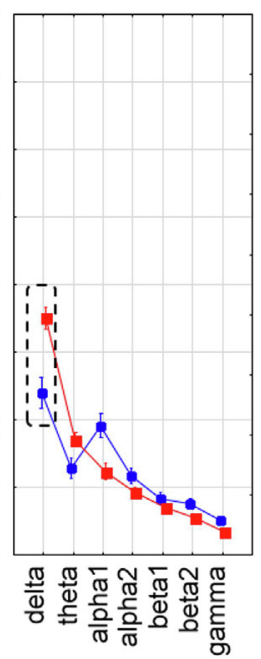

Central

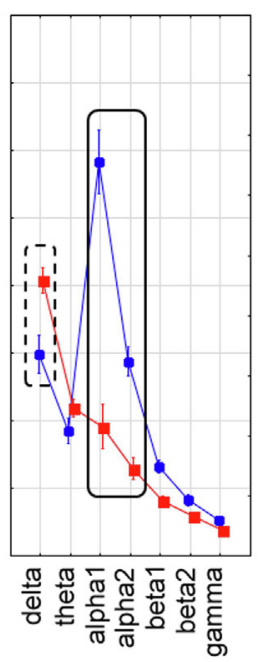

Parietal

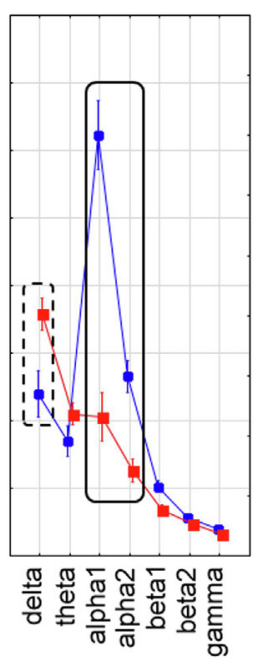

Occipital

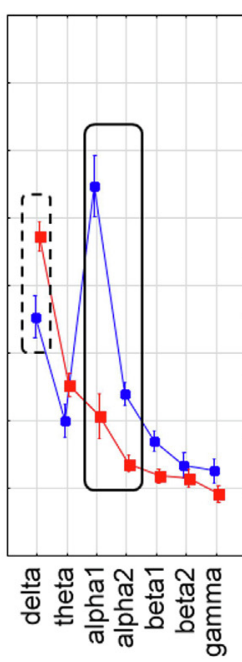

Temporal

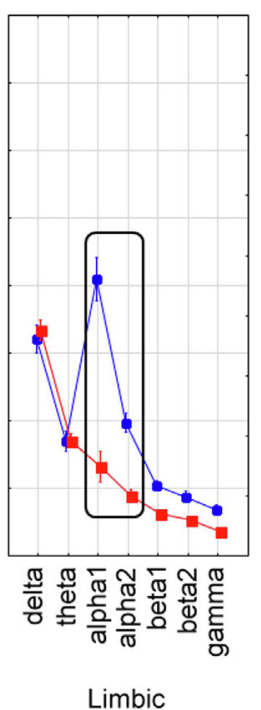

Limbic

c Nold $=$ ADD

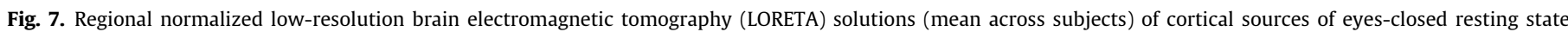

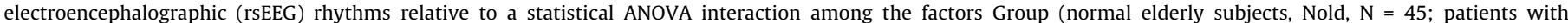

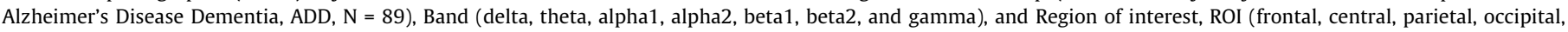

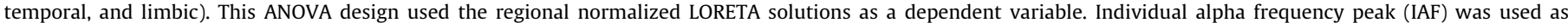

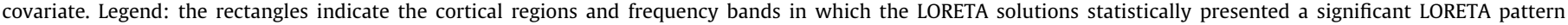
Nold $\neq$ ADD (Bonferroni corrected $p<0.05$; the Bonferroni correction was applied for 7 frequency bands X 6 ROIs $=42, p<0.05 / 42=0.001$ ).

\section{Table 6}

Results of the sMRI part of the control analysis between normal elderly subjects (Nold, $\mathrm{N}=45$ ) and patients with Alzheimer's Disease Dementia (ADD, $\mathrm{N}=89$ ). Rankbased nonparametric analysis (Kruskal-Wallis $\mathrm{H}$ test) was used to test the differences between sMRI variables in both group of ADD and Nold. The results show sMRI variables have statistically significant differences ( $p<0.01$ to 0.001 ).

\begin{tabular}{ll}
\hline Structural MRI biomarkers & $\begin{array}{l}\text { Statistical analysis (Kruskal-Wallis H } \\
\text { test; Nold }>\text { ADD) }\end{array}$ \\
\hline $\begin{array}{l}\text { Gray matter volume normalized } \\
\text { by TIV }\end{array}$ & $X^{2}(1)=30.8, p=0.001$ \\
White matter volume & $X^{2}(1)=12.6, p=0.001$ \\
Cerebral fluid under the skull & $X^{2}(1)=10.3, p=0.001$ \\
Left hippocampus volume & $X^{2}(1)=22.2, p=0.001$ \\
$\begin{array}{l}\text { Left hippocampus volume } \\
\text { normalized by TIV }\end{array}$ & $X^{2}(1)=26.8, p=0.001$ \\
$\begin{array}{l}\text { Right hippocampus volume } \\
\text { Right hippocampus volume } \\
\text { normalized by TIV }\end{array}$ & $X^{2}(1)=26.8, p=0.001$ \\
$\begin{array}{l}\text { Total hippocampus volume } \\
\text { Total hippocampus volume } \\
\text { normalized by TIV }\end{array}$ & $X^{2}(1)=23.6, p=0.001$ \\
$\begin{array}{l}\text { Total hippocampus volume } \\
\text { normalized by GM }\end{array}$ & $X^{2}(1)=23.2, p=0.001$ \\
$\begin{array}{l}\text { Cerebral cortex volume } \\
\text { Cerebral cortex volume } \\
\text { normalized by TIV }\end{array}$ & $X^{2}(1)=27.5, p=0.001$ \\
$\begin{array}{c}\text { Cerebral cortex volume } \\
\text { normalized by GM }\end{array}$ & $X^{2}(1)=3.9, p=0.046$ \\
& $X^{2}(1)=33.5, p=0.001$ \\
& $X^{2}(1)=3.9, p=0.046$
\end{tabular}

TIV: Total Intracranial Volume; GM: gray matter; CSF: Cerebrospinal fluid; sMRI: structural magnetic resonance imaging.

papers. In of them (Jonkman, 1997) the accuracy of the classification of the Nold and AD subjects ranged from $54 \%$ to $100 \%$ while the other article (Jelic and Kowalski, 2009) reported accuracy of about $80-85 \%$ between the ADD or $\mathrm{MCI}$ patients and individuals with other forms of dementing disorders.

Another interesting finding of this research is that the ANNs with SAEs allowed a moderate classification accuracy with rsEEG
(80\%) just slightly lower than the accuracy obtained with the sMRI features (84\%). Furthermore, rsEEG and sMRI features were nonredundant to each other, as their combination gave the highest classification accuracy (89\%). The same conclusions were reached adopting a different architecture where a pair of AEs was specialized to recognize either ADD or Nold related input patterns. These results enrich the complex picture of the previous multimodal classification studies using rsEEG and sMRI in AD patients. In this research line, Colloby et al. (2016) showed that the classification using rsEEG spectral features had sensitivity of $87 \%$ in the detection of ADD patients and $62 \%$ in the detection of DLB patients, even better than the respective sensitivity values of $77 \%$ and $52 \%$ based on sMRI features. Combining rsEEG and sMRI features, Colloby et al. (2016) reached the highest sensitivity of 93\% for ADD patients and $86 \%$ for DLB patients. In the same vein, Polikar et al. (2010) reported that combined EEG, sMRI, and FDG-PET features showed that the accuracy of classification between Nold and ADD individuals improved up to $10-20 \%$ compared with the outcome of the single modalities. Moreover, Fernández et al. (2003) reported that combined sMRI and rsEEG features pointed to the highest accuracy of $87 \%$ in the same classification task. Analogously, Prieto Del Val et al. (2016) reached the best classification accuracy of about $80 \%$ (sensitivity $78 \%$ and specificity $82 \%$ ) between Nold and ADD individuals using combined rsEEG and sMRI features.

An original finding of this study is that the ANNs with SAEs unveiled high values of sensitivity (89\%) in the detection of ADD individuals, with a less performant specificity in recognition of Nold subjects (64\%). This sensitivity was higher than that obtained using resilient backpropagation ANNs in our reference study of Triggiani et al. (2017), which exhibited a sensitivity of $79 \%$ and specificity of $74 \%$. The high sensitivity in the detection of ADD individuals may make the present ANN procedure of interest for clinical applications as a "gatekeeper" of seniors at risk of ADD to undergo more complex and expensive diagnostic procedures such as PET mapping of brain amyloid accumulation or the invasive 
lumbar puncture for the dosing of $A \beta 42$ and phospho-tau proteins (Jack et al, 2018). Indeed, EEG techniques are non-invasive, costeffective, easily accessible, and replicable in long longitudinal studies (Jelic and Kowalski, 2009). According to this "gatekeeper" function, seniors with "ADD-like" rsEEG markers may undergo more invasive and expensive diagnostic procedures such as PET mapping of brain amyloid accumulation or lumbar puncture for the dosing of A 342 and phospho-tau proteins (Dubois et al., 2014; Jack et al., 2018). In this perspective, the relatively low specificity of the present ANN procedure would result in some Nold subjects "ADD-like" undergoing CSF and neuroimaging diagnostic procedures. This may be a reasonable cost when compared to general population screening programs in seniors based on CSF and neuroimaging diagnostic procedures.

Finally, it is essential to acknowledge some important limitations of the present study, which needs to be replicated in independent cohorts of patients with more advanced procedures. In the present study, we used the traditional clinical 10-20 montage with 19 scalp exploring electrodes (e.g., 10-20 system), which is not ideal for an optimal spatial sampling of rsEEG rhythms aimed at estimating underlying cortical sources (Liu et al., 2002). Indeed, previous evidence showed that EEG source estimates based on few scalp exploring electrodes led to both blurring of source estimates and incorrect spatial localizations (Michel and Brunet, 2019). These estimation errors may be fatal in the localization of circumscribed sources of epileptiform activity from < 32 scalp exploring electrodes, especially in addition to undetected errors in the placement of electrodes placed over the scalp (Brinkmann et al., 1998; Lantz et al., 2003; Michel and Brunet, 2019). Moreover, a further cause of approximation in the present rsEEG source estimation was the use of the standard LORETA cortical model (i.e., MNI152 developed by Montreal Neurologic Institute) for all participants rather than the use of realistic head models based on their individual MRIs. Those models would have much better accounted for the inter-individual variability of cortical mantles.

In the present explorative study, we partially mitigated these methodological limitations averaging normalized LORETA source estimates within bilateral lobar ROIs rather than to perform a localization study at the level of single voxels or small ROIs considered in each hemisphere separately. The present EEG source estimation at bilateral lobar ROIs may represent an acceptable approximation for widespread maps of cortical rsEEG rhythms expected during a condition of quiet wakefulness keeping the eyes closed in a silent room (Babiloni et al., 2020a,b). Indeed, the LORETA toolbox was designed to estimate widespread EEG source patterns fitting large distributions of scalp EEG activity (PascualMarqui et al., 1994), as its mathematical regularization procedure identifies maximally smoothed source current solutions at the cortical level explaining scalp-recorded potential distributions (Halder et al., 2019). Cortical rsEEG rhythms may have those ideal features for LORETA source estimations.

\section{Conclusions}

In the present retrospective and exploratory study, we used ANNs with two architectures (i.e., SAE and AE) of stacked (autoencoding) hidden layers recreating input data in the output, to examine the classification accuracy between the Nold and ADD individuals based on input features derived from sMRI, rsEEG, and a combination of both variables. The feature database referred to Nold and ADD individuals enrolled by several clinical units in different clinical trials.

Results showed that the ANN with stacked autoencoders and a deep leaning model representing both ADD and control participants (i.e., SAE) showed classification accuracies in discriminating them of $80 \%, 85 \%$, and $89 \%$ using rsEEG, sMRI, and rsEEG + sMRI features, respectively. The two ANNs with stacked autoencoders and a deep leaning model specialized for either ADD or control participants (i.e., AE) showed classification accuracies of $77 \%, 83 \%$, and $86 \%$ using the same input features.

These findings confirmed the general robustness of this kind of ANNs for the present application and motivate future resource investments and use of more advanced procedures in this research line. Those procedures may include 1 ) the use of $>48-64$ exploring electrodes and the digitization of their true position on the scalp; 2 ) the use of individual head models for rsEEG source estimations at small ROIs localized in each hemisphere to gain spatial detail; 3) a fine-grain individual analysis of frequency bands based on IAFp (Klimesch, 1999); 4) a parallel frequency analysis considering the entire rsEEG power spectrum as a unified statistical representation of the EEG signal that may take into account age-related 1 /f noise distribution, which may cause a flattening of the EEG power spectrum (Voytek et al., 2015); 5) ensuring future studies use consistent EEG and MRI data collection methods across all participants; and 6) the exploration of further variants of the present stacked autoencoder ANNs and other reference machine learning techniques (e.g., K nearest neighbors, support vector machines, random forests or random decision forests, etc.).

If the best combination of those procedures allowed the best classification accuracy in the discrimination between Nold and ADD individuals, they may be applied for research purposes in the classification between Nold seniors and ADMCI patients and the detection of ADMCI patients with probable rapid trajectories of progression to dementia.

It should be remarked that even if the above scenarios were successful, the present ANNs could not be directly translated into clinical applications. A tentative roadmap for clinical translations may imply at least the following steps: 1) cross-validation with extended specificity as a replication of the current model of ANN classification in a fully independent rsEEG and MRI database collected not only in Nold and ADD individuals but also patients with dementing disorders typically misdiagnosed as ADD such as dementia with Lewy bodies (Rizzo et al., 2018) and limbicpredominant age-related TDP-43 encephalopathy (Nelson et al., 2019); 2) establishment of the particular combinations of rsEEGSMRI patterns that could be clinically relevant for detection of ADD patients; and 3) definition of a consensus about rsEEG/sMRI recording guidelines and open-access platforms with toolboxes for a friendly semi-automatic derivation of significant and informative rsEEG/sMRI biomarkers and use of the validated ANNs providing the probability that a senior with cognitive deficits under examination might suffer from $\mathrm{AD}$ or have a rapid clinical trajectory.

\section{Funding}

This study was partially supported by a fund from the Italian Ministry of Health "Ricerca Corrente" (RC n. 2751586) (Dr. Ferri). The research activities of the Unit of University of Rome "La Sapienza" were partially supported by the H2020 Marie S. Curie ITN-ETN project with the short title "BBDiag" (http://bbdiag-itnetn.eu) and the H2020-TWINN-2015 project with the short title "SynaNet" (https://www.synanet2020.com).

\section{Declaration of Competing Interest}

The authors declare that they have no known competing financial interests or personal relationships that could have appeared to influence the work reported in this paper. 


\section{References}

Adler G, Brassen S, Jajcevic A. EEG coherence in Alzheimer's dementia. J Neural Transm 2003;110:1051-8. https://doi.org/10.1007/s00702-003-0024-8.

Albert MS, DeKosky ST, Dickson D, Dubois B, Feldman HH, Fox NC, et al. The diagnosis of mild cognitive impairment due to Alzheimer's disease: Recommendations from the National Institute on Aging-Alzheimer's Association workgroups on diagnostic guidelines for Alzheimer's disease. Alzheimers Dement 2011;7:270-9. https://doi.org/10.1016/ j.jalz.2011.03.008.

Babiloni C, Brancucci A, Capotosto P, Romani GL, Arendt-Nielsen L, Chen AC, et al. Slow cortical potential shifts preceding sensorimotor interactions. Brain Res Bull 2005;65:309-16. https://doi.org/10.1016/i.brainresbull.2004.11.023.

Babiloni C, Brancucci A, Vecchio F, Arendt-Nielsen L, Chen AC, Rossini PM. Anticipation of somatosensory and motor events increases centro-parietal functional coupling: an EEG coherence study. Clin Neurophysiol 2006;117:1000-8. https://doi.org/10.1016/i.clinph.2005.12.028.

Babiloni C, Lizio R, Carducci F, Vecchio F, Redolfi A, Marino S, et al. Resting state cortical electroencephalographic rhythms and white matter vascular lesions in subjects with Alzheimer's disease: an Italian multicenter study. J Alzheimers Dis 2011;26:331-46. https://doi.org/10.3233/JAD-2011-101710.

Babiloni C, Del Percio C, Bordet R, Bourriez JL, Bentivoglio M, Payoux P, et al. Effects of acetylcholinesterase inhibitors and memantine on resting-state electroencephalographic rhythms in Alzheimer's disease patients. Clin Neurophysiol 2013;124:837-50. https://doi.org/10.1016/i.clinph.2012.09.017.

Babiloni C, Del Percio C, Boccardi M, Lizio R, Lopez S, Carducci F, et al. Occipital sources of resting-state alpha rhythms are related to local gray matter density in subjects with amnesic mild cognitive impairment and Alzheimer's disease. Neurobiol Aging 2015;36:556-70. https://doi.org/10.1016/i. neurobiolaging.2014.09.011.

Babiloni C, Triggiani AI, Lizio R, Cordone S, Tattoli G, Bevilacqua V, et al. Classification of single normal and Alzheimer's disease individuals from cortical sources of resting state EEG rhythms. Front Neurosci 2016;10:47. https://doi.org/10.3389/fnins.2016.00047.

Babiloni C, Del Percio C, Lizio R, Noce G, Cordone S, Lopez S, et al. Abnormalities of cortical neural synchronization mechanisms in patients with dementia due to Alzheimer's and Lewy body diseases: an EEG study. Neurobiol Aging 2017:55:143-58. https://doi.org/10.1016/i.neurobiolaging.2017.03.030.

Babiloni C, Del Percio C, Lizio R, Noce G, Lopez S, Soricelli A, et al. Abnormalities of resting state cortical EEG rhythms in subjects with mild cognitive impairment due to Alzheimer's and Lewy body diseases. J Alzheimers Dis 2018;62:247-68. https://doi.org/10.3233/JAD-170703.

Babiloni C, Blinowska K, Bonanni L, Cichocki A, De Haan W, Del Percio C, et al. What electrophysiology tells us about Alzheimer's disease: a window into the synchronization and connectivity of brain neurons. Neurobiol Aging 2020; 85: 58-73. 10.1016/j.neurobiolaging.2019.09.008

Babiloni C, Barry RJ, Başar E, Blinowska KJ, Cichocki A, Drinkenburg WHIM, et al. International Federation of Clinical Neurophysiology (IFCN) - EEG research workgroup: Recommendations on frequency and topographic analysis of resting state EEG rhythms. Part 1: Applications in clinical research studies. Clin Neurophysiol 2020b;131:285-307. https://doi.org/10.1016/ j.clinph.2019.06.234.

Besthorn C, Zerfass R, Geiger-Kabisch C, Sattel H, Daniel S, Schreiter-Gasser U, et al. Discrimination of Alzheimer's disease and normal aging by EEG data. Electroencephalogr Clin Neurophysiol 1997;103:241-8. https://doi.org/ 10.1016/s0013-4694(97)96562-7.

Braak H, Braak E. Staging of Alzheimer's disease-related neurofibrillary changes. Neurobiol Aging 1995;16:271-8. https://doi.org/10.1016/0197-4580(95) 00021-6.

Brenner RP, Ulrich RF, Spiker DG, Sclabassi RJ, Reynolds 3rd CF, Marin RS, et al. Computerized EEG spectral analysis in elderly normal, demented and depressed subjects. Electroencephalogr Clin Neurophysiol 1986;64:483-92. https://doi. org/10.1016/0013-4694(86)90184-7.

Brenner RP, Reynolds 3rd CF, Ulrich RF. Diagnostic efficacy of computerized spectral versus visual EEG analysis in elderly normal, demented and depressed subjects. Electroencephalogr Clin Neurophysiol 1988;69:110-7. https://doi.org/10.1016/ 0013-4694(88)90206-4.

Brinkmann BH, O'Brien TJ, Dresner MA, Lagerlund TD, Sharbrough FW, Robb RA. Scalp-recorded EEG localization in MRI volume data. Brain Topogr 1998;10:245-53. https://doi.org/10.1023/a:1022266822252.

Burgess RC. Filtering of neurophysiologic signals. Handb Clin Neurol 2019;160:51-65. https://doi.org/10.1016/B978-0-444-64032-1.00004-7.

Chiaramonti R, Muscas GC, Paganini M, Müller TJ, Fallgatter AJ, Versari A, et al. Correlations of topographical EEG features with clinical severity in mild and moderate dementia of Alzheimer type. Neuropsychobiology 1997;36:153-8. https://doi.org/10.1159/000119375.

Claus JJ, Strijers RL, Jonkman EJ, Ongerboer de Visser BW, Jonker C, Walstra GJ, et al. The diagnostic value of electroencephalography in mild senile Alzheimer's disease. Clin Neurophysiol 1999;110:825-32. https://doi.org/10.1016/s13882457(98)00076-5.

Clinical and neuropathological criteria for frontotemporal dementia. The Lund and Manchester Groups. J Neurol Neurosurg Psychiatry 1994; 57: 416-8. 10.1136/ jnnp.57.4.416.

Colloby SJ, Cromarty RA, Peraza LR, Johnsen K, Jóhannesson G, Bonanni L, et al. Multimodal EEG-MRI in the differential diagnosis of Alzheimer's disease and dementia with Lewy bodies. J Psychiatr Res 2016;78:48-55. https://doi.org/ 10.1016/j.jpsychires.2016.03.010.

Dierks T, Ihl R, Frölich L, Maurer K. Dementia of the Alzheimer type: effects on the spontaneous EEG described by dipole sources. Psychiatry Res 1993;50:151-62. https://doi.org/10.1016/0925-4927(93)90027-F.

Dierks T, Jelic V, Pascual-Marqui RD, Wahlund L, Julin P, Linden DE, et al. Spatial pattern of cerebral glucose metabolism (PET) correlates with localization of intracerebral EEG-generators in Alzheimer's disease. Clin Neurophysiol 2000;111:1817-24. https://doi.org/10.1016/S1388-2457(00)00427-2.

Dubois B, Feldman HH, Jacova C, Hampel H, Molinuevo JL, Blennow K, et al. Advancing research diagnostic criteria for Alzheimer's disease: the IWG-2 criteria. Lancet Neurol 2014;13:614-29. https://doi.org/10.1016/S1474-4422 (14)70090-0.

Engedal K, Snaedal J, Hoegh P, Jelic V, Bo Andersen B, Naik M, et al. Quantitative EEG applying the statistical recognition pattern method: a useful tool in dementia diagnostic workup. Dement Geriatr Cogn Disord 2015;40:1-12. https://doi.org 10.1159/000381016.

Fernández A, Arrazola J, Maestú F, Amo C, Gil-Gregorio P, Wienbruch C, et al. Correlations of hippocampal atrophy and focal low-frequency magnetic activity in Alzheimer disease: volumetric MR imaging-magnetoencephalographic study. AJNR Am J Neuroradiol 2003;24:481-7.

Folstein MF, Folstein SE, McHugh PR. "Mini-mental state". A practical method for grading the cognitive state of patients for the clinician. J Psychiatr Res 1975; 12 : 189-98. 0022-3956(75)90026-6.

Förstl H, Kurz A. Clinical features of Alzheimer's disease. Eur Arch Psychiatry Clin Neurosci 1999;249:288-90. https://doi.org/10.1007/s004060050101. PMID: 10653284.

Gelb DJ, Oliver E, Gilman S. Diagnostic criteria for Parkinson disease. Arch Neurol 1999;56:33-9. https://doi.org/10.1001/archneur.56.1.33.

Halder T, Talwar S, Jaiswal AK, Banerjee A. Quantitative evaluation in estimating sources underlying brain oscillations using current source density methods and beamformer approaches. eNeuro 2019; 6:ENEURO.0170-19.2019. 10.1523/ ENEURO.0170-19.2019.

Hooijer C, Jonker C, Posthuma J, Visser SL. Reliability, validity and follow-up of the EEG in senile dementia: sequelae of sequential measurement. Electroencephalogr Clin Neurophysiol 1990;76:400-12. https://doi.org/ 10.1016/0013-4694(90)90094-z.

Huang C, Wahlund L, Dierks T, Julin P, Winblad B, Jelic V. Discrimination of Alzheimer's disease and mild cognitive impairment by equivalent EEG sources: a cross-sectional and longitudinal study. Clin Neurophysiol 2000;111:1961-7. https://doi.org/10.1016/S1388-2457(00)00454-5.

Hughes CP, Berg L, Danziger WL, Coben LA, Martin RL. A new clinical scale for the staging of dementia. Br J Psychiatry 1982;140:566-72. https://doi.org/10.1192/ bjp.140.6.566.

Jack Jr CR, Knopman DS, Jagust WJ, Shaw LM, Aisen PS, Weiner MW, et al. Hypothetical model of dynamic biomarkers of the Alzheimer's pathological cascade. Lancet Neurol 2010;9:119-28. https://doi.org/10.1016/S1474-4422 (09)70299-6.

Jack CR Jr, Bennett DA, Blennow K, Carrillo MC, Dunn B, Haeberlein SB, et al. NIA-AA Research Framework: Toward a biological definition of Alzheimer's disease. Alzheimers Dement 2018; 14: 535-562. 10.1016/j.jalz.2018.02.018.

Jelic V, Shigeta M, Julin P, Almkvist O, Winblad B, Wahlund LO. Quantitative electroencephalography power and coherence in Alzheimer's disease and mild cognitive impairment. Dementia 1996;7:314-23. https://doi.org/10.1159/ 000106897.

Jelic V, Kowalski J. Evidence-based evaluation of diagnostic accuracy of resting EEG in dementia and mild cognitive impairment. Clin EEG Neurosci 2009;40:129-42. https://doi.org/10.1177/155005940904000211.

Jeong J. EEG dynamics in patients with Alzheimer's disease. Clin Neurophysiol 2004;115:1490-505. https://doi.org/10.1016/j.clinph.2004.01.001.

Jicha G. Topographical markers of Alzheimer's disease Pprogression: Can MRI and FDG-PET serve as surrogate outcomes in clinical trials of disease modifying agents in Alzheimer's disease?. Alzheimers Dement 2011;7:S1. https://doi.org/ 10.1016/i.jalz.2011.05.2431.

Jonkman EJ. The role of the electroencephalogram in the diagnosis of dementia of the Alzheimer type: an attempt at technology assessment. Neurophysiol Clin 1997;27:211-9. https://doi.org/10.1016/S0987-7053(97)83777-X.

Karami V, Nittari G, Amenta F. Neuroimaging computer-aided diagnosis systems for Alzheimer's disease. Int J Imaging Syst Technol 2018:1-12. https://doi.org/ 10.1002/ima. 22300.

Klimesch W. EEG alpha and theta oscillations reflect cognitive and memory performance: a review and analysis. Brain Res Brain Res Rev 1999; 29: 169-95. 10.1016/s0165-0173(98)00056-3.

Lantz G, Grave de Peralta R, Spinelli L, Seeck M, Michel CM. Epileptic source localization with high density EEG: how many electrodes are needed? Clin Neurophysiol 2003; 114: 63-9. 10.1016/s1388-2457(02)00337-1.

Larochelle H, Bengio Y, Louradour J, Lamblin P. Exploring strategies for training deep neural networks. J Machine Learn Res 2009;10:1-40.

Lawrence S, Giles CL. Overfitting and neural networks: conjugate gradient and backpropagation. Proceedings of the IEEE-INNS-ENNS International Joint Conference on Neural Networks. IJCNN 2000. Neural Computing: New Challenges and Perspectives for the New Millennium, Como, Italy 2000; 1 : 114-119. 10.1109/IJCNN.2000.857823.

Lawton MP, Brod EM. Assessment of older people: self-maintaining and instrumental activities of daily living. Gerontologist 1969;9:179-86. https:/ doi.org/10.1093/geront/9.3_Part 1.179. 
Lee S, Lee H, Kim KW. Alzheimer's Disease Neuroimaging Initiative. Magnetic resonance imaging texture predicts progression to dementia due to Alzheimer disease earlier than hippocampal volume. J Psychiatry Neurosci 2020;45:7-14. https://doi.org/10.1503/ipn.180171.

Leuchter AF, Cook IA, Newton TF, Dunkin J, Walter DO, Rosenberg-Thompson S, et al. Regional differences in brain electrical activity in dementia: use of spectral power and spectral ratio measures. Electroencephalogr Clin Neurophysiol 1993;87:385-93. https://doi.org/10.1016/0013-4694(93)90152-l. PMID: 7508371.

Liao B, Xu J, Lv J, Zhou S. An image retrieval method for binary images based on DBN and softmax classifier. IETE Tech Rev 2015; 32: 294-303. 10.1080/ 02564602.2015 .1015631$.

Liu AK, Dale AM, Belliveau JW. Monte Carlo simulation studies of EEG and MEG localization accuracy. Hum Brain Mapp 2002;16:47-62. https://doi.org/ 10.1002/hbm.10024.

Lizio R, Del Percio C, Marzano N, Soricelli A, Yener GG, Başar E, et al. Neurophysiological assessment of Alzheimer's disease individuals by a single electroencephalographic marker. J Alzheimers Dis 2016;49:159-77. https://doi. org/10.3233/IAD-143042.

McKeith IG, Dickson DW, Lowe J, Emre M, O’Brien JT, Feldman H, et al. Diagnosis and management of dementia with Lewy bodies: third report of the DLB Consortium. Neurology 2005; 65: 1863-72. 10.1212/01. wnl.0000187889.17253.b1.

McKhann G, Drachman D, Folstein M, Katzman R, Price D, Stadlan EM. Clinical diagnosis of Alzheimer's disease: report of the NINCDS-ADRDA Work Group under the auspices of Department of Health and Human Services Task Force on Alzheimer's Disease. Neurology 1984;34:939-44. https://doi.org/10.1212/ WNL.34.7.939.

McKhann GM, Knopman DS, Chertkow H, Hyman BT, Jack Jr CR, Kawas CH, et al. The diagnosis of dementia due to Alzheimer's disease: recommendations from the National Institute on Aging-Alzheimer's Association workgroups on diagnostic guidelines for Alzheimer's disease. Alzheimers Dement 2011;7:263-9. https:/ doi.org/10.1016/j.jalz.2011.03.005.

Michel CM, Brunet D. EEG source imaging: A practical review of the analysis steps. Front Neurol 2019;10:325. https://doi.org/10.3389/fneur.2019.00325.

Møller MF. A scaled conjugate gradient algorithm for fast supervised learning. Neural Networks 1993;6:525-33. https://doi.org/10.1016/S0893-6080(05) 80056-5.

Moretti DV, Babiloni F, Carducci F, Cincotti F, Remondini E, Rossini PM, et al. Computerized processing of EEG-EOG-EMG artifacts for multi-centric studies in EEG oscillations and event-related potentials. Int $\mathrm{J}$ Psychophysiol 2003;47:199-216. https://doi.org/10.1016/S0167-8760(02)00153-8.

Moretti DV, Frisoni GB, Fracassi C, Pievani M, Geroldi C, Binetti G, et al. MCI patients' EEGs show group differences between those who progress and those who do not progress to AD. Neurobiol Aging 2011;32:563-71. https://doi.org/10.1016/i. neurobiolaging.2009.04.003.

Nelson PT, Dickson DW, Trojanowski JQ, Jack CR, Boyle PA, Arfanakis K, et al. Limbicpredominant age-related TDP-43 encephalopathy (LATE): consensus working group report. Brain 2019;142:1503-27. https://doi.org/10.1093/brain/awz099.

Nuwer M. Assessment of digital EEG, quantitative EEG, and EEG brain mapping: report of the American Academy of Neurology and the American Clinical Neurophysiology Society. Neurology 1997;49:277-92. https://doi.org/10.1212/ wnl.49. 1.277 .

Ottoy J, Niemantsverdriet E, Verhaeghe J, De Roeck E, Struyfs H, Somers C, et al. Association of short-term cognitive decline and MCI-to-AD dementia conversion with CSF, MRI, amyloid- and 18F-FDG-PET imaging. Neuroimage Clin 2019;22. https://doi.org/10.1016/i.nicl.2019.101771 101771.

Pascual-Marqui RD, Michel CM, Lehmann D. Low resolution electromagnetic tomography: a new method for localizing electrical activity in the brain. Int J $\begin{array}{lll}\text { Psychophysiol 1994;18:49-65. } & \text { https://doi.org/10.1016/j. }\end{array}$ ineumeth.2006.10.023.

Polikar R, Tilley C, Hillis B, Clark CM. Multimodal EEG, MRI and PET data fusion for Alzheimer's disease diagnosis. Annu Int Conf IEEE Eng Med Biol Soc 2010; 2010: 6058-61. 10.1109/IEMBS.2010.5627621.
Ponomareva NV, Selesneva ND, Jarikov GA. EEG alterations in subjects at high familial risk for Alzheimer's disease. Neuropsychobiology 2003;48:152-9. https://doi.org/10.1159/000073633.

Prieto Del Val L, Cantero JL, Atienza M. Atrophy of amygdala and abnormal memoryrelated alpha oscillations over posterior cingulate predict conversion to Alzheimer's disease. Sci Rep 2016;6:31859. https://doi.org/10.1038/srep31859.

Román GC, Tatemichi TK, Erkinjuntti T, Cummings JL, Masdeu JC, Garcia JH, et al. Vascular dementia: diagnostic criteria for research studies. Report of the NINDS-AIREN International Workshop. Neurology 1993; 43: 250-60. 10.1212/ WNL.43.2.250.

Rizzo G, Arcuti S, Copetti M, Alessandria M, Savica R, Fontana A, et al. Accuracy of clinical diagnosis of dementia with Lewy bodies: a systematic review and metaanalysis. J Neurol Neurosurg Psychiatry 2018;89:358-66.

Rossini PM, Di Iorio R, Vecchio F, Anfossi M, Babiloni C, Bozzali M, et al. Early diagnosis of Alzheimer's disease: the role of biomarkers including advanced EEG signal analysis. Report from the IFCN-sponsored panel of experts. Clin Neurophysiol 2020;131:1287-310.

Sperling RA, Aisen PS, Beckett LA, Bennett DA, Craft S, Fagan AM, et al. Toward defining the preclinical stages of Alzheimer's disease: recommendations from the National Institute on Aging-Alzheimer's Association workgroups on diagnostic guidelines for Alzheimer's disease. Alzheimers Dement 2011;7:280-92. https://doi.org/10.1016/i.jalz.2011.03.003.

Strijers RL, Scheltens P, Jonkman EJ, de Rijke W, Hooijer C, Jonker C. Diagnosing Alzheimer's disease in community-dwelling elderly: a comparison of EEG and MRI. Dement Geriatr Cogn Disord 1997;8:198-202. https://doi.org/10.1159/ $\underline{000106631 .}$.

Suk HI, Lee SW, Shen D; Alzheimer's Disease Neuroimaging Initiative. Latent feature representation with stacked auto-encoder for AD/MCI diagnosis. Brain Struct Funct 2015; 220: 841-59. 10.1007/s00429-013-0687-3.

Talairach J, Tournoux P. Co-planar Stereotaxic Atlas of the human brain. New York: Thieme Medical Publishers; 1988.

Tzourio-Mazoyer N, Landeau B, Papathanassiou D, Crivello F, Etard O, Delcroix N, et al. Automated anatomical labeling of activations in SPM using a macroscopic anatomical parcellation of the MNI MRI single-subject brain. Neuroimage 2002;15:273-89. https://doi.org/10.1006/nimg.2001.0978.

Trambaiolli LR, Falk TH, Fraga FJ, Anghinah R, Lorena AC. EEG spectro-temporal modulation energy: a new feature for automated diagnosis of Alzheimer's disease. Annu Int Conf IEEE Eng Med Biol Soc 2011;2011:3828-31. https://doi. org/10.1109/IEMBS.2011.6090951.

Triggiani AI, Bevilacqua V, Brunetti A, Lizio R, Tattoli G, Cassano F, et al. Classification of healthy subjects and Alzheimer's disease patients with dementia from cortical sources of resting state EEG rhythms: a study using artificial neural networks. Front Neurosci 2017;10:604. https://doi.org/10.3389/ fnins.2016.00604.

Voytek B, Kramer MA, Case J, Lepage KQ, Tempesta ZR, Knight RT, et al. Age-related changes in 1/f neural electrophysiological noise. J Neurosci 2015;35:13257-65. https://doi.org/10.1523/INEUROSCI.2332-14.2015.

Weiner MW, Veitch DP, Aisen PS, Beckett LA, Cairns NJ, Green RC, et al. Alzheimer's disease neuroimaging initiative. Recent publications from the Alzheimer's disease neuroimaging initiative: reviewing progress toward improved $A D$ clinical trials. Alzheimers Dement 2017;13:e1-e85. https://doi.org/10.1016/ j.jalz.2016.11.007.

Willis-Shattuck M, Bidwell P, Thomas S, Wyness L, Blaauw D, Ditlopo P. Motivation and retention of health workers in developing countries: a systematic review. BMC Health Serv Res 2008;8:247. https://doi.org/10.1186/1472-6963-8-247.

Yesavage JA, Brink TL, Rose TL, Lum O, Huang V, Adey M, et al. Development and validation of a geriatric depression screening scale: a preliminary report. J Psychiatr Res 1982-1983;17:37-49. https://doi.org/10.1016/0022-3956(82) 90033-4.

Zhang Y, Brady M, Smith S. Segmentation of brain MR images through a hidden Markov random field model and the expectation-maximization algorithm. IEEE Trans Med Imaging 2001;20:45-57. https://doi.org/10.1109/42.906424. 